\title{
On far-outlying constant mean curvature spheres in asymptotically flat Riemannian 3-manifolds
}

\author{
By Otis Chodosh at Princeton and Michael Eichmair at Vienna
}

\begin{abstract}
We extend the Lyapunov-Schmidt analysis of outlying stable constant mean curvature spheres in the work of S. Brendle and the second-named author [3] to the "far-offcenter" regime and to include general Schwarzschild asymptotics. We obtain sharp existence and non-existence results for large stable constant mean curvature spheres that depend delicately on the behavior of scalar curvature at infinity.
\end{abstract}

\section{Introduction}

We complement in this paper our recent work [5] on the characterization of the leaves of the canonical foliation as the unique large closed embedded stable constant mean curvature surfaces in strongly asymptotically flat Riemannian 3-manifolds. More precisely, we extend here the Lyapunov-Schmidt analysis of outlying stable constant mean curvature spheres developed by S. Brendle and the second-named author in [3] to include the far-off-center regime and general Schwarzschild asymptotics.

We begin by introducing some standard notation.

Throughout this paper, we consider complete Riemannian 3-manifolds $(M, g)$ so there are both a compact set $K \subset M$ and a diffeomorphism

$$
M \backslash K \cong\left\{x \in \mathbb{R}^{3}:|x|>\frac{1}{2}\right\}
$$

such that, in this chart at infinity, for some $q>\frac{1}{2}$ and non-negative integer $k$,

$$
g_{i j}=\delta_{i j}+\tau_{i j}
$$

where

$$
\partial_{I} \tau_{i j}=O\left(|x|^{-q-|I|}\right)
$$

for all multi-indices $I$ of length $|I| \leq k$. Moreover, we require that the boundary $\partial M$ of $M$, if non-empty, is a minimal surface and such that the components of $\partial M$ are the only connected closed minimal surfaces in $(M, g)$. We say that $(M, g)$ is $C^{k}$-asymptotically flat of rate $q$.

Otis Chodosh was partially supported by an NSF grant DMS-1811059. Michael Eichmair was partially supported by the FWF START-Project Y963-N35.

(๕)BY (C) 2019 Otis Chodosh and Michael Eichmair, published by De Gruyter. This work is licensed under the Creative Commons Attribution 4.0 Public License. 
It is convenient to denote, for $r>1$, by $S_{r}$ the surface in $M$ that corresponds to the centered coordinate sphere $S_{r}(0)=\left\{x \in \mathbb{R}^{3}:|x|=r\right\}$ and by $B_{r}$ the bounded open region enclosed by $S_{r}$ and $\partial M$. Given $A \subset M$, we let

$$
r_{0}(A):=\sup \left\{r>1: A \cap B_{r}=\emptyset\right\} .
$$

A particularly important example of an asymptotically flat Riemannian 3-manifold is Schwarzschild initial data

$$
M=\left\{x \in \mathbb{R}^{3}:|x| \geq \frac{m}{2}\right\} \quad \text { and } \quad g=\left(1+\frac{m}{2|x|}\right)^{4} \sum_{i=1}^{3} d x^{i} \otimes d x^{i},
$$

where $m>0$ is the mass parameter.

We say that $(M, g)$ as above is $C^{k}$-asymptotic to Schwarzschild of mass $m>0$ if, instead of (1.1), we have

$$
g_{i j}=\left(1+\frac{m}{2|x|}\right)^{4} \delta_{i j}+\sigma_{i j}
$$

where

$$
\partial_{I} \sigma_{i j}=O\left(|x|^{-2-|I|}\right)
$$

for all multi-indices $I$ of length $|I| \leq k$.

Our contributions here when combined with the key result in [5] give the following theorem.

Theorem 1.1 ([5]). Suppose that $(M, g)$ is a complete Riemannian 3-manifold that is $C^{6}$-asymptotic to Schwarzschild of mass $m>0$ and whose scalar curvature vanishes. Every connected closed embedded stable constant mean curvature surface with sufficiently large area is a leaf of the canonical foliation.

The canonical foliation $\left\{\Sigma_{H}\right\}_{0<H<H_{0}}$ of $M \backslash K$ (for appropriate $H_{0}>0$ and $K \subset M$ compact) through stable constant mean curvature spheres $\Sigma_{H}$ with respective mean curvature $H$ was discovered by G. Huisken and S.-T. Yau in [9]. They showed that, for every $s \in\left(\frac{1}{2}, 1\right]$, there is $H_{S} \in\left(0, H_{0}\right)$ such that for $H \in\left(0, H_{S}\right), \Sigma_{H}$ is the only stable constant mean curvature sphere of mean curvature $H$ in $(M, g)$ that encloses the ball $\left\{x \in \mathbb{R}^{3}:|x|<H^{-s}\right\}$ in the chart at infinity. This characterization was later refined by J. Qing and G. Tian [13]: Every leaf $\Sigma_{H}$ of the canonical foliation is the unique stable constant mean curvature sphere of mean curvature $H$ in $(M, g)$ that encloses $K$. (In [5, Appendix F] we provide an alternative argument of this result from [13] in the case where the scalar curvature of $(M, g)$ is non-negative.) In joint work with A. Carlotto [4] and inspired by earlier work of J. Metzger and the second-named author [6], we extended this characterization further under the additional assumption that the scalar curvature of $(M, g)$ is non-negative in the following way: Choose a point $p \in M$. Every connected stable constant mean curvature sphere $\Sigma \subset M$ that encloses $p$ and whose area is sufficiently large is a leaf of the canonical foliation. Thus, to prove an unconditional uniqueness result along the lines of Theorem 1.1, it remains to understand large stable constant mean curvature spheres that are outlying in the sense that the bounded region they enclose is disjoint and - in view of the results in [4] - far from $K$. The center of mass flux integrals used in $[9,13]$ vanish in this regime; new ideas are needed. S. Brendle and the second-named author discovered a subtle relationship between scalar curvature and outlying stable constant mean curvature 
spheres in [3]. They gave examples of divergent sequences $\left\{\Sigma_{k}\right\}_{k=1}^{\infty}$ of outlying stable constant mean curvature spheres in $(M, g)$ asymptotic to Schwarzschild with $m>0$, which is the setting of $[9,13]$. In fact, $\Sigma_{k}$ is a perturbation of the coordinate sphere

$$
S_{\lambda_{k}}\left(\lambda_{k} \xi\right)=\left\{\left|x-\lambda_{k} \xi\right|=\lambda_{k}: x \in \mathbb{R}^{3}\right\}
$$

in the chart at infinity, where $\xi \in \mathbb{R}^{3}$ is such that $|\xi|>1$ and where $\lambda_{k} \rightarrow \infty$. On the other hand, they showed that no such sequences can exist in $(M, g)$ if the scalar curvature is nonnegative, provided a further technical assumption on the expansion of the metric in the chart at infinity holds.

Theorem 1.2 (S. Brendle and M. Eichmair [3]). Let $(M, g)$ be a complete Riemannian 3-manifold that is $C^{4}$-asymptotic to Schwarzschild with mass $m>0$. In addition to (1.2), we also require here that

$$
g_{i j}=\left(1+\frac{m}{2|x|}\right)^{4} \delta_{i j}+T_{i j}+o\left(|x|^{-2}\right) \quad \text { as }|x| \rightarrow \infty,
$$

where $T_{i j}$ is homogeneous of degree -2, with corresponding estimates for all partial derivatives of order $\leq 4$. Assume that the scalar curvature of $g$ satisfies $R \geq-o\left(|x|^{-4}\right)$ as $|x| \rightarrow \infty$. There does not exist a sequence $\left\{\Sigma_{k}\right\}_{k=1}^{\infty}$ of outlying closed stable constant mean curvature surfaces in $(M, g)$ with

$$
r_{0}\left(\Sigma_{k}\right) \rightarrow \infty \text { and } r_{0}\left(\Sigma_{k}\right) H\left(\Sigma_{k}\right) \rightarrow \eta>0 .
$$

In the statement of this result and below, we use $H\left(\Sigma_{k}\right)$ to denote the constant mean curvature of $\Sigma_{k}$ with respect to the outward pointing unit normal in $(M, g)$. Our sign convention is such that coordinate spheres in Euclidean space have positive mean curvature.

In our recent work [5], we show that when $(M, g)$ is asymptotic to Schwarzschild with mass $m>0$ and if the scalar curvature is non-negative, there are no sequences $\left\{\Sigma_{k}\right\}_{k=1}^{\infty}$ of embedded stable constant mean curvature spheres in $(M, g)$ with

$$
r_{0}\left(\Sigma_{k}\right) \rightarrow \infty \text { and } r_{0}\left(\Sigma_{k}\right) H\left(\Sigma_{k}\right) \rightarrow 0 .
$$

(Note that the assumptions here imply that $H\left(\Sigma_{k}\right) \rightarrow 0$ and thus that area $g\left(\Sigma_{k}\right) \rightarrow \infty$.) Assuming in addition that $g$ has additional homogeneity as in Theorem 1.2, this leaves only the scenario of sequences $\left\{\Sigma_{k}\right\}_{k=1}^{\infty}$, where

$$
r_{0}\left(\Sigma_{k}\right) \rightarrow \infty, \quad \operatorname{area}_{g}\left(\Sigma_{k}\right) \rightarrow \infty, \quad r_{0}\left(\Sigma_{k}\right) H\left(\Sigma_{k}\right) \rightarrow \infty
$$

to fully understand large stable constant mean curvature spheres in $(M, g)$. To study this final scenario and to investigate whether the assumption of additional homogeneity in the expansion of the metric (1.3) off of Schwarzschild in Theorem 1.2 is really necessary, we revisit in this paper the Lyapunov-Schmidt analysis carried out in [3].

We show that Theorem 1.2 is false without the assumption of additional homogeneity in the expansion of the metric.

Theorem 1.3. There is an asymptotically flat complete Riemannian 3-manifold $(M, g)$ with non-negative scalar curvature that is smoothly asymptotic to Schwarzschild of mass $m>0$ in the sense that

$$
g_{i j}=\left(1+\frac{m}{2|x|}\right)^{4} \delta_{i j}+\sigma_{i j},
$$


where

$$
\partial_{I} \sigma_{i j}=O\left(|x|^{-2-|I|}\right)
$$

for all multi-indices $I$, and which contains a sequence $\left\{\Sigma_{k}\right\}_{k=1}^{\infty}$ of outlying stable constant mean curvature spheres with

$$
r_{0}\left(\Sigma_{k}\right) \rightarrow \infty \text { and } r_{0}\left(\Sigma_{k}\right) H\left(\Sigma_{k}\right) \rightarrow \eta>0 .
$$

We also prove that we may drop the assumption of additional homogeneity of the metric in Theorem 1.2 in favor of a mild growth condition on the scalar curvature.

Theorem 1.4. Let $(M, g)$ be a complete Riemannian 3-manifold that is $C^{4}$-asymptotic to Schwarzschild in the sense that

$$
g_{i j}=\left(1+\frac{m}{2|x|}\right)^{4} \delta_{i j}+\sigma_{i j},
$$

where $\partial_{I} \sigma_{i j}=O\left(|x|^{-2-|I|}\right)$ for all multi-indices $I$ of length $|I| \leq 4$. We also assume that either

$$
R=o\left(|x|^{-4}\right) \quad \text { as }|x| \rightarrow \infty
$$

or

$$
x^{i} \partial_{i}\left(|x|^{2} R\right) \leq o\left(|x|^{-2}\right) \quad \text { as }|x| \rightarrow \infty .
$$

There does not exist a sequence $\left\{\Sigma_{k}\right\}_{k=1}^{\infty}$ of closed outlying stable constant mean curvature surfaces in $(M, g)$ with

$$
r_{0}\left(\Sigma_{k}\right) \rightarrow \infty \text { and } r_{0}\left(\Sigma_{k}\right) H\left(\Sigma_{k}\right) \rightarrow \eta>0 .
$$

Note that (1.5) holds in either one of the following two cases.

(i) When $R=0$. This is for example the case when $(M, g)$ is time symmetric initial data for a vacuum spacetime.

(ii) When the metric in the chart at infinity has the special form (1.3) in Theorem 1.2, then

$$
R=S+o\left(|x|^{-4}\right)
$$

where

$$
S=\sum_{i, j=1}^{3}\left(\partial_{i} \partial_{j} T_{i j}-\partial_{i} \partial_{i} T_{j j}\right) .
$$

Note that $S$ is homogeneous of degree -4 . Euler's theorem shows that (1.5) holds if and only if $R \geq-o\left(|x|^{-4}\right)$. Thus Theorem 1.4 generalizes Theorem 1.2 to the nonhomogeneous setting.

It is interesting to compare (1.5) to condition (H3) in S. Brendle's version of Alexandrov's theorem for certain warped products [1]. Note that our example constructed in the proof of Theorem 1.3 is a warped product. We also mention that $\mathrm{S}$. Ma has constructed examples of $(M, g)$ that contain large unstable constant mean curvature spheres [10]. The scalar curvature in these examples is negative in some places; see [10, discussion preceding the statement of Theorem 1.1 and proof of Lemma 4.7]. 
We now turn to the case of large stable constant mean curvature spheres that are very far outlying in the sense that

$$
r_{0}\left(\Sigma_{k}\right) \rightarrow \infty, \quad \operatorname{area}_{g}\left(\Sigma_{k}\right) \rightarrow \infty, \quad r_{0}\left(\Sigma_{k}\right) H\left(\Sigma_{k}\right) \rightarrow \infty .
$$

These surfaces are not within the scope of the Lyapunov-Schmidt analysis in [3], where case (1.4) is considered. The main difficulty in the regime considered here is that the "Schwarzschild contribution" to the reduced area functional leveraged in [3] is no longer on the order of $O(1)$ but instead decaying. To proceed, it is necessary to obtain rather involved estimates for the reduced functional. To describe our results, we first recall the basic setup and some terminology from [3] that we will also adopt here. A standard application of the implicit function theorem shows that, given $\lambda>0$ large and $\xi \in \mathbb{R}^{3}$ with $|\xi|>1$, there is a closed surface $\Sigma_{(\xi, \lambda)}$ in the chart at infinity so that the following hold:

- $\Sigma_{(\xi, \lambda)}$ bounds volume $\frac{4 \pi \lambda^{3}}{3}$ with respect to the metric $g$.

- $\Sigma_{(\xi, \lambda)}$ is the Euclidean graph of a function $u_{(\xi, \lambda)}$ on $S_{\lambda}(\lambda \xi)$, i.e.

$$
\Sigma_{(\xi, \lambda)}=\left\{\lambda \xi+y+\frac{u_{(\xi, \lambda)}(x) y}{\lambda}: x=\lambda \xi+y \in S_{\lambda}(\lambda \xi)\right\},
$$

where

$$
\sup _{S_{\lambda}(\lambda \xi)}\left|u_{(\xi, \lambda)}\right|+\lambda \sup _{S_{\lambda}(\lambda \xi)}\left|\nabla u_{(\xi, \lambda)}\right|+\lambda^{2} \sup _{S_{\lambda}(\lambda \xi)}\left|\nabla^{2} u_{(\xi, \lambda)}\right|=O\left(\frac{1}{|\xi|}\right) .
$$

- $u_{(\xi, \lambda)}$ is orthogonal to the first spherical harmonics on $S_{\lambda}(\lambda \xi)$ with respect to the Euclidean metric.

- The mean curvature of $\Sigma_{(\xi, \lambda)}$ with respect to $g$ viewed as a function on $S_{\lambda}(\lambda \xi)$ is the restriction of a linear function.

Given a sequence $\left\{\Sigma_{k}\right\}_{k=1}^{\infty}$ of connected closed stable constant mean curvature surfaces with

$$
r_{0}\left(\Sigma_{k}\right) \rightarrow \infty, \quad \operatorname{area}_{g}\left(\Sigma_{k}\right) \rightarrow \infty, \quad r_{0}\left(\Sigma_{k}\right) H\left(\Sigma_{k}\right) \rightarrow \infty,
$$

the argument in [3, p. 676] shows that for all $k$ large enough, $\Sigma_{k}=\Sigma_{\left(\xi_{k}, \lambda_{k}\right)}$ for appropriate $\lambda_{k}>0$ and $\xi_{k} \in \mathbb{R}^{3}$ both large. Whether $(M, g)$ admits such sequences can now be decided using the following result.

Theorem 1.5. Let $(M, g)$ be a complete Riemannian 3-manifold that is $C^{5+\ell}$-asymptotic to Schwarzschild with mass $m=2$, where $\ell$ is a non-negative integer. Let $\lambda>0$ and $\xi \in \mathbb{R}^{3}$ both be large. Then

$$
\begin{array}{r}
\operatorname{area}_{g}\left(\Sigma_{(\xi, \lambda)}\right)=4 \pi \lambda^{2}-\frac{2 \pi}{15} \lambda^{4} R(\lambda \xi)-\frac{\pi}{105} \lambda^{6}(\Delta R)(\lambda \xi) \\
-\frac{8 \pi}{35}|\xi|^{-6}+O\left(\lambda^{-1}|\xi|^{-6}\right)+O\left(|\xi|^{-7}\right)
\end{array}
$$

where $R$ is the scalar curvature of $(M, g) .{ }^{1)}$ This expansion can be differentiated $\ell$ times with respect to $\xi$.

1) We may compute the Laplacian of scalar curvature either with respect to $g$ or with respect to the Euclidean background metric in the chart at infinity. The difference is of the order of the error terms. 
Let $\ell \geq 1$. As in [12] or [3], we use that the map

$$
\xi \mapsto \operatorname{area}_{g}\left(\Sigma_{(\xi, \lambda)}\right)
$$

has a critical point at $\xi$ if, and only if, $\Sigma_{(\xi, \lambda)}$ is a constant mean curvature sphere. If $\ell \geq 2$, then the critical point is stable if, and only if, $\Sigma_{(\xi, \lambda)}$ is a stable constant mean curvature sphere. This immediately leads to the following corollary.

Corollary 1.6. Let $(M, g)$ be a complete Riemannian 3-manifold that is $C^{6}$-asymptotic to Schwarzschild in the sense that

$$
g_{i j}=\left(1+\frac{m}{2|x|}\right)^{4} \delta_{i j}+\sigma_{i j},
$$

where $\partial_{I} \sigma_{i j}=O\left(|x|^{-2-|I|}\right)$ for all multi-indices $I$ of length $|I| \leq 6$. Assume that the scalar curvature vanishes. There does not exist a sequence $\left\{\Sigma_{k}\right\}_{k=1}^{\infty}$ of connected closed stable constant mean curvature surfaces in $(M, g)$ with

$$
r_{0}\left(\Sigma_{k}\right) \rightarrow \infty, \quad \operatorname{area}_{g}\left(\Sigma_{k}\right) \rightarrow \infty, \quad \text { and } \quad r_{0}\left(\Sigma_{k}\right) H\left(\Sigma_{k}\right) \rightarrow \infty
$$

The analogue of Theorem 1.4 in this setting is not so clear-cut. We have the following result.

Corollary 1.7. Let $(M, g)$ be a complete Riemannian 3-manifold that is $C^{7}$-asymptotic to Schwarzschild in the sense that

$$
g_{i j}=\left(1+\frac{m}{2|x|}\right)^{4} \delta_{i j}+\sigma_{i j}
$$

where

$$
\partial_{I} \sigma_{i j}=O\left(|x|^{-2-|I|}\right)
$$

for all multi-indices I of length $|I| \leq 6$. We also assume that the scalar curvature $R$ of $(M, g)$ is radially convex at infinity in the sense that

$$
x^{i} x^{j} \partial_{i} \partial_{j} R \geq 0
$$

outside of a compact set. There does not exist a sequence $\left\{\Sigma_{k}\right\}_{k=1}^{\infty}$ of connected closed stable constant mean curvature surfaces in $(M, g)$ with

$$
r_{0}\left(\Sigma_{k}\right) \rightarrow \infty, \quad \operatorname{areag}_{g}\left(\Sigma_{k}\right) \rightarrow \infty, \quad r_{0}\left(\Sigma_{k}\right) H\left(\Sigma_{k}\right) \rightarrow \infty
$$

Our assumption (1.7) here is surprisingly sharp. Comparing with Theorem 1.2 or Theorem 1.4, one might be lead to conjecture that it can be weakened to

(i) assuming that $x^{i} x^{j} \partial_{i} \partial_{j} R \geq-o\left(|x|^{-4}\right)$ as $|x| \rightarrow \infty$, or, more generally,

(ii) assuming that $\sigma_{i j}=T_{i j}+o\left(|x|^{-2}\right)$ as $|x| \rightarrow \infty$ where $T_{i j}$ homogeneous of order -2 , and that the scalar curvature is non-negative.

The following example dashes any hope of such generalizations. 
Theorem 1.8. There is an asymptotically flat complete Riemannian 3-manifold $(M, g)$ with non-negative scalar curvature such that, in the chart at infinity,

$$
g_{i j}=\left(1+|x|^{-1}\right)^{4} \delta_{i j}+T_{i j}+o\left(|x|^{-4}\right) \quad \text { as }|x| \rightarrow \infty
$$

along with all derivatives, where $T_{i j}$ is homogeneous of degree -2, and which contains a sequence $\left\{\Sigma_{k}\right\}_{k=1}^{\infty}$ of outlying stable constant mean curvature spheres with

$$
r_{0}\left(\Sigma_{k}\right) \rightarrow \infty, \quad \operatorname{area} g\left(\Sigma_{k}\right) \rightarrow \infty, \quad r_{0}\left(\Sigma_{k}\right) H\left(\Sigma_{k}\right) \rightarrow \infty .
$$

To conclude, we note that Lyapunov-Schmidt analysis was used by, e.g., R. Ye [14], S. Nardulli [11], and F. Pacard and X. Xu in [12] to study the related question of when small geodesic spheres admit perturbations to constant mean curvature. The answer depends on the scalar curvature. In particular, S. Nardulli [11] applied Lyapunov-Schmidt reduction to study the expansion of the isoperimetric profile of a Riemannian manifold for small volumes. We also note that there is a large body of work on stable constant mean curvature spheres in general asymptotically flat Riemannian 3-manifolds. We refer the reader to [5, Section 2.1] for an overview and references to results in this direction.

Acknowledgement. We thank S. Brendle for helpful conversations.

\section{Proof of Theorem 1.4}

The proof follows the lines of [3] with one important difference: We do not assume here that the deviation of the metric from Schwarzschild is homogeneous of degree -2 to top order. Without loss of generality, we may assume that the mass $m$ is equal to 2 . Thus,

$$
g_{i j}=\left(1+|x|^{-1}\right)^{4} \delta_{i j}+\sigma_{i j}
$$

where

$$
\partial_{I} \sigma_{i j}=O\left(|x|^{-2-|I|}\right)
$$

for all multi-indices $I$ of length $|I| \leq 4$.

Let $\Omega$ be a bounded subset with compact closure in $\mathbb{R}^{3} \backslash \overline{B_{1}(0)}$. For $\xi \in \Omega$ and $\lambda>0$ sufficiently large, we may use the implicit function theorem to find surfaces $\Sigma_{(\xi, \lambda)}$ as in [3, Proposition 4]. Moreover, the surface $\Sigma_{(\xi, \lambda)}$ is a constant mean curvature sphere (respectively, a stable constant mean curvature sphere) if, and only if, $\xi$ is a critical point (respectively, a stable critical point) for the map $\xi \mapsto \operatorname{area}_{g}\left(\Sigma_{(\xi, \lambda)}\right)$. The derivation of [3, Proposition 5] carries over to give

$$
\operatorname{area}_{g}\left(\Sigma_{(\xi, \lambda)}\right)=4 \pi \lambda^{2}+2 \pi F_{0}(|\xi|)+F_{\sigma}(\xi, \lambda)+o(1) \quad \text { as } \lambda \rightarrow \infty .
$$

Indeed, the assumption that $\sigma$ is homogeneous is not used up to this point of [3]. We recall that

$$
F_{0}(t)=-14+16 t^{2} \log \frac{t^{2}-1}{t^{2}}+\left(15 t-t^{-1}\right) \log \frac{t+1}{t-1}
$$

is the contribution from the Schwarzschild background, while

$$
F_{\sigma}(\xi, \lambda)=\frac{1}{2} \int_{S_{(\xi, \lambda)}} \operatorname{tr}_{S_{(\xi, \lambda)}} \sigma-\frac{1}{\lambda} \int_{B_{(\xi, \lambda)}} \operatorname{tr} \sigma
$$

is the contribution from $\sigma$. 
Here and below, unless explicitly noted otherwise, all geometric operations are with respect to the Euclidean background metric in the chart at infinity. Following the notation of [3], given $\xi \in \mathbb{R}^{3}$ and $\lambda>0$, we will often write

$$
S_{(\xi, \lambda)}=S_{\lambda}(\lambda \xi) \quad \text { and } \quad B_{(\xi, \lambda)}=B_{\lambda}(\lambda \xi)
$$

2.1. Radial variation. In the computation of the radial derivative of (2.2) in [3, proof of Proposition 7], the additional assumption that $\sigma$ is homogeneous is used in various places. We compute the radial derivative of (2.2) for general $\sigma$ below. Our computation parallels that in [3] but requires several additional integrations by part:

$$
\begin{aligned}
& \left(\nabla_{\xi} F_{\sigma}\right)(\xi, \lambda)=\left.\frac{d}{d s}\right|_{s=1} F_{\sigma}(s \xi, \lambda) \\
& \left.=\frac{\lambda}{2} \int_{S_{(\xi, \lambda)}} \operatorname{tr}_{S_{(\xi, \lambda)}} \nabla_{\xi} \sigma-\int_{S_{(\xi, \lambda)}}(\operatorname{tr} \sigma)\langle\xi, \nu\rangle \quad \text { (we write } \xi=\xi^{\top}+\langle\xi, \nu\rangle v\right) \\
& =\frac{\lambda}{2} \int_{S_{(\xi, \lambda)}}\left(\operatorname{tr}_{S_{(\xi, \lambda)}} \nabla_{\nu} \sigma\right)\langle\xi, \nu\rangle-\int_{S_{(\xi, \lambda)}}(\operatorname{tr} \sigma)\langle\xi, \nu\rangle \\
& +\frac{\lambda}{2} \int_{S_{(\xi, \lambda)}} \operatorname{tr}_{S_{(\xi, \lambda)}} \nabla_{\xi} \sigma \\
& =\frac{\lambda}{2} \int_{S_{(\xi, \lambda)}}\left(\operatorname{tr}_{S_{(\xi, \lambda)}} \nabla_{\nu} \sigma\right)\langle\xi, \nu\rangle-\int_{S_{(\xi, \lambda)}}(\operatorname{tr} \sigma)\langle\xi, \nu\rangle \\
& +\frac{\lambda}{2} \int_{S_{(\xi, \lambda)}} \nabla_{\xi} \operatorname{tr} \sigma-\left(\nabla_{\xi} \sigma\right)(v, v) \\
& =\frac{\lambda}{2} \int_{S_{(\xi, \lambda)}}\left(\operatorname{tr}_{S_{(\xi, \lambda)}} \nabla_{\nu} \sigma\right)\langle\xi, \nu\rangle-\int_{S_{(\xi, \lambda)}}(\operatorname{tr} \sigma)\langle\xi, \nu\rangle \\
& +\frac{\lambda}{2} \int_{S_{(\xi, \lambda)}} \nabla_{\xi}(\operatorname{tr} \sigma-\sigma(v, v))+2 \sigma\left(\nabla_{\xi} v, v\right) \\
& =\frac{\lambda}{2} \int_{S_{(\xi, \lambda)}}\left(\operatorname{tr}_{S_{(\xi, \lambda)}} \nabla_{\nu} \sigma\right)\langle\xi, \nu\rangle-\int_{S_{(\xi, \lambda)}}(\operatorname{tr} \sigma)\langle\xi, \nu\rangle \\
& +\frac{\lambda}{2} \int_{S_{(\xi, \lambda)}}\left((\operatorname{tr} \sigma-\sigma(v, v))\left(-\operatorname{div}_{S_{(\xi, \lambda)}} \xi^{\top}\right)+2 \sigma\left(\nabla_{\xi} \top v, v\right)\right. \\
& =\frac{\lambda}{2} \int_{S_{(\xi, \lambda)}}\left(\operatorname{tr}_{S_{(\xi, \lambda)}} \nabla_{\nu} \sigma\right)\langle\xi, \nu\rangle-\int_{S_{(\xi, \lambda)}}(\operatorname{tr} \sigma)\langle\xi, \nu\rangle \\
& +\int_{S_{(\xi, \lambda)}}(\operatorname{tr} \sigma)\langle\xi, \nu\rangle-\sigma(v, v)\langle\xi, v\rangle+\sigma\left(\xi^{\top}, v\right) \\
& =\frac{\lambda}{2} \int_{S_{(\xi, \lambda)}}\left(\operatorname{tr}_{S_{(\xi, \lambda)}} \nabla_{\nu} \sigma\right)\langle\xi, v\rangle+\int_{S_{(\xi, \lambda)}} \sigma(\xi, \nu)-2 \sigma(v, v)\langle\xi, v\rangle .
\end{aligned}
$$

We define a vector field

$$
Y=\langle\xi, v\rangle \sigma(\nu, \cdot)^{\#}
$$

on $S_{(\xi, \lambda)}$ and compute

$$
\operatorname{div}_{S_{(\xi, \lambda)}} Y=\frac{1}{\lambda} \sigma(\xi, v)-\frac{1}{\lambda}\langle\xi, v\rangle \sigma(\nu, v)+\langle\xi, \nu\rangle \operatorname{tr}_{S_{(\xi, \lambda)}}(\nabla . \sigma)(v, \cdot)+\frac{1}{\lambda}\langle\xi, v\rangle \operatorname{tr}_{S_{(\xi, \lambda)}} \sigma .
$$


The first variation formula gives

$\frac{\lambda}{2} \int_{S_{(\xi, \lambda)}}\left(\operatorname{tr}_{S_{(\xi, \lambda)}}(\nabla . \sigma)(\nu, \cdot)\right)\langle\xi, \nu\rangle=\frac{1}{2} \int_{S_{(\xi, \lambda)}}\left(3 \sigma(\nu, \nu)-\operatorname{tr}_{S_{(\xi, \lambda)}} \sigma\right)\langle\xi, \nu\rangle-\frac{1}{2} \int_{S_{(\xi, \lambda)}} \sigma(\xi, \nu)$.

We insert this into the above expression, and continue:

$$
\begin{aligned}
\left.\frac{d}{d s}\right|_{s=1} F(s \xi, \lambda)= & \frac{\lambda}{2} \int_{S_{(\xi, \lambda)}}\left(\operatorname{tr}_{S_{(\xi, \lambda)}} \nabla_{\nu} \sigma-\operatorname{tr}_{S_{(\xi, \lambda)}}(\nabla \cdot \sigma)(\nu, \cdot)\right)\langle\xi, \nu\rangle \\
& -\frac{1}{2} \int_{S_{(\xi, \lambda)}}(\operatorname{tr} \sigma)\langle\xi, \nu\rangle-\sigma(\xi, \nu) .
\end{aligned}
$$

We write $\langle\xi, v\rangle=-|\xi|^{2}+\lambda^{-1}\langle\xi, X\rangle$ in the first integrand, where $X$ is the position field, to obtain

$$
\begin{aligned}
\left.\frac{d}{d s}\right|_{s=1} F(s \xi, \lambda)= & \frac{\lambda}{2} \int_{S_{(\xi, \lambda)}}\left(\operatorname{tr}_{S_{(\xi, \lambda)}}(\nabla . \sigma)(\nu, \cdot)-\operatorname{tr}_{S_{(\xi, \lambda)}} \nabla_{\nu} \sigma\right)\left(|\xi|^{2}-\lambda^{-1}\langle\xi, X\rangle\right) \\
& -\frac{1}{2} \int_{S_{(\xi, \lambda)}}(\operatorname{tr} \sigma)\langle\xi, \nu\rangle-\sigma(\xi, \nu) \\
= & \frac{\lambda}{2} \int_{S_{(\xi, \lambda)}}\left(\operatorname{tr}(\nabla . \sigma)(\nu, \cdot)-\operatorname{tr} \nabla_{\nu} \sigma\right)\left(|\xi|^{2}-\lambda^{-1}\langle\xi, X\rangle\right) \\
& -\frac{1}{2} \int_{S_{(\xi, \lambda)}}(\operatorname{tr} \sigma)\langle\xi, \nu\rangle-\sigma(\xi, \nu) .
\end{aligned}
$$

We define a vector field $W=\operatorname{div} \sigma-\nabla \operatorname{tr} \sigma$. This yields

$$
\begin{aligned}
\left.\frac{d}{d s}\right|_{s=1} F(s \xi, \lambda)= & \frac{1}{2} \int_{S_{(\xi, \lambda)}}\langle\xi, \lambda \xi-X\rangle\langle W, v\rangle-\frac{1}{2} \int_{S_{(\xi, \lambda)}}(\operatorname{tr} \sigma)\langle\xi, v\rangle-\sigma(\xi, \nu) \\
= & \frac{1}{2} \int_{B_{(\xi, \lambda)}} \operatorname{div}(\langle\xi, \lambda \xi-X\rangle W)-\frac{1}{2} \int_{S_{(\xi, \lambda)}}(\operatorname{tr} \sigma)\langle\xi, v\rangle-\sigma(\xi, \nu) \\
= & \frac{1}{2} \int_{B_{(\xi, \lambda)}}(\operatorname{div} W)\langle\xi, \lambda \xi-X\rangle-\frac{1}{2} \int_{B_{(\xi, \lambda)}}\langle\xi, W\rangle \\
& \quad-\frac{1}{2} \int_{S_{(\xi, \lambda)}}(\operatorname{tr} \sigma)\langle\xi, \nu\rangle-\sigma(\xi, v) .
\end{aligned}
$$

Note that $\langle\xi, W\rangle=\operatorname{div}(\sigma(\xi, \cdot)-(\operatorname{tr} \sigma) \xi)$. We apply the divergence theorem to arrive at

$$
\begin{aligned}
\left.\frac{d}{d s}\right|_{s=1} F(s \xi, \lambda)= & \frac{1}{2} \int_{B_{(\xi, \lambda)}}(\operatorname{div} W)\langle\xi, \lambda \xi-X\rangle-\frac{1}{2} \int_{S_{(\xi, \lambda)}} \sigma(\xi, v)-(\operatorname{tr} \sigma)\langle\xi, v\rangle \\
& \quad-\frac{1}{2} \int_{S_{(\xi, \lambda)}}(\operatorname{tr} \sigma)\langle\xi, v\rangle-\sigma(\xi, \nu) \\
= & \frac{1}{2} \int_{B_{(\xi, \lambda)}}(\operatorname{div} W)\langle\xi, \lambda \xi-X\rangle .
\end{aligned}
$$

Note that

$$
\operatorname{div} W=R+O\left(|x|^{-5}\right),
$$

where $R$ is the scalar curvature of $g$. In conclusion, we obtain

(2.3) $\left(\nabla_{\xi} F_{\sigma}\right)(\xi, \lambda)=\left.\frac{d}{d s}\right|_{s=1} F(s \xi, \lambda)=\frac{1}{2} \int_{B_{(\xi, \lambda)}}\langle\xi, \lambda \xi-X\rangle R+o(1) \quad$ as $\lambda \rightarrow \infty$. 
2.2. Radial variation in spherical coordinates. Assume first that

$$
R \geq 0 \quad \text { and } \quad x^{i} \partial_{i}\left(|x|^{2} R\right) \leq 0 .
$$

For definiteness, we assume that

$$
\xi=|\xi| e_{3},
$$

where $|\xi|>1$. In this subsection, we compute the radial variation

$$
\int_{B_{\lambda}(\lambda \xi)}\langle\lambda \xi-X, \xi\rangle R
$$

in spherical coordinates

$$
(\rho, \phi, \theta) \mapsto(\rho \sin \phi \cos \theta, \rho \sin \phi \sin \theta, \rho \cos \phi) .
$$

on the complement of the $z$-axis. The radial line in direction

$$
(\sin \phi \cos \theta, \sin \phi \sin \theta, \cos \phi)
$$

intersects the ball $B_{\lambda}(\lambda \xi)$ in the $\rho$-interval whose endpoints are the solutions

$$
\rho_{ \pm}=\lambda|\xi|\left(\cos \phi \pm\left(\frac{1}{|\xi|^{2}}-\sin ^{2} \phi\right)^{\frac{1}{2}}\right)
$$

of the quadratic equation

$$
\rho^{2}-2 \rho \lambda|\xi| \cos \phi+\lambda^{2}\left(|\xi|^{2}-1\right)=0
$$

The intersection is non-empty for angles $\phi \in\left[0, \phi_{+}\right]$, where $\phi_{+} \in(0, \pi)$ solves

$$
\sin ^{2} \phi_{+}=\frac{1}{|\xi|^{2}} \text {. }
$$

We then have that

$$
\begin{aligned}
& \int_{B_{\lambda}(\lambda \xi)}\langle\lambda \xi-X, \xi\rangle R \\
& \quad=\int_{0}^{2 \pi} \int_{0}^{\phi_{+}} \int_{\rho_{-}}^{\rho_{+}} R(\rho, \phi, \theta)\left(\lambda|\xi|^{2}-\rho|\xi| \cos \phi\right) \rho^{2} \sin \phi d \rho d \phi d \theta \\
& \quad=|\xi| \int_{0}^{2 \pi} \int_{0}^{\phi_{+}} \int_{\rho_{-}}^{\rho_{+}} \rho^{2} R(\rho, \phi, \theta)(\lambda|\xi|-\rho \cos \phi) \sin \phi d \rho d \phi d \theta \\
& \quad \geq|\xi| \int_{0}^{2 \pi} \int_{0}^{\phi_{+}}\left(\frac{\lambda|\xi|}{\cos \phi}\right)^{2} R\left(\frac{\lambda|\xi|}{\cos \phi}, \phi, \theta\right)\left(\int_{\rho_{-}}^{\rho_{+}}(\lambda|\xi|-\rho \cos \phi) d \rho\right) \sin \phi d \phi d \theta .
\end{aligned}
$$

Now, for every $\phi \in\left(0, \phi_{+}\right)$,

$$
\int_{\rho_{-}}^{\rho_{+}}(\lambda|\xi|-\rho \cos \phi) d \rho=\left(\rho_{+}-\rho_{-}\right) \lambda|\xi| \sin ^{2} \phi>0
$$

so that, in conclusion,

$$
\int_{B_{\lambda}(\lambda \xi)}\langle\lambda \xi-X, \xi\rangle R \geq 0
$$

Arguing as in [3, p. 677] shows that $\Sigma_{(\xi, \lambda)}$ cannot be a constant mean curvature sphere. 
We now discuss how to proceed if we only impose the weaker assumption (1.5). Note that $R=O\left(|x|^{-4}\right)$ by asymptotic flatness. It follows that there is a function $f$ with $f=o\left(|x|^{-4}\right)$ as $|x| \rightarrow \infty$ such that

$$
R+f \geq 0 \quad \text { and } \quad x^{i} \partial_{i}\left(|x|^{2}(R+f)\right) \leq 0 .
$$

Proceeding as before, we obtain the estimate

$$
\int_{B_{\lambda}(\lambda \xi)}\langle\lambda \xi-X, \xi\rangle R \geq-o(1) \quad \text { as } \lambda \rightarrow \infty .
$$

This is enough for the argument in [3] to yield that $\Sigma_{(\xi, \lambda)}$ does not have constant mean curvature.

\section{Proof of Theorem 1.3}

We follow the lines of [3, proof of Theorem 1] in that our metric has a pulse in its scalar curvature which forces the reduced area functional $\xi \mapsto \operatorname{area}_{g}\left(\Sigma_{(\xi, \lambda)}\right)$ to have stable critical points. Unlike the examples in [3], our metric is spherically symmetric and - more importantly - has non-negative scalar curvature.

Let $S:(0, \infty) \rightarrow(-\infty, 0]$ be a smooth function such that

$$
S^{(\ell)}(r)=O\left(r^{-4-\ell}\right)
$$

for every non-negative integer $\ell$. We define a smooth function $\varphi:(0, \infty) \rightarrow \mathbb{R}$ by

$$
\varphi(r)=\frac{1}{r} \int_{r}^{\infty}(\rho-r) \rho S(\rho) d \rho .
$$

Note that

$$
\varphi^{\prime}(r)=-\frac{1}{r^{2}} \int_{r}^{\infty} \rho^{2} S(\rho) d \rho
$$

so

$$
\frac{\left(r^{2} \varphi^{\prime}\right)^{\prime}}{r^{2}}=S(r)
$$

Lemma 3.1. For every non-negative integer $\ell$, we have that

$$
\varphi^{(\ell)}(r)=O\left(r^{-2-\ell}\right) .
$$

Proof. Because $S(r)=O\left(r^{-4}\right)$, we see that

$$
\varphi(r)=O\left(r^{-2}\right) \quad \text { and } \quad \varphi^{\prime}(r)=O\left(r^{-3}\right) .
$$

Using (3.1), we find

$$
\varphi^{\prime \prime}(r)+\frac{2 \varphi^{\prime}(r)}{r}=S(r) .
$$

From this, the asserted decay of the higher derivatives can be verified by induction. 
On $\mathbb{R}^{3} \backslash\{0\}$, we define a conformally flat Riemannian metric

$$
g=\left(1+\frac{1}{r}+\varphi(r)\right)^{4} \bar{g}=\left(1+\frac{1}{r}\right)^{4} \bar{g}+O\left(\frac{1}{r^{2}}\right),
$$

where $r=|x|$. Note that $g$ is smoothly asymptotic to Schwarzschild with mass 2. Its scalar curvature is easily computed as

$$
R=-8\left(1+\frac{1}{r}+\varphi(r)\right)^{-5} \frac{\left(r^{2} \varphi^{\prime}\right)^{\prime}}{r^{2}}=-8\left(1+O\left(\frac{1}{r}\right)\right) S(r) .
$$

In particular, the scalar curvature is non-negative on the complement of a compact set. We now make a particular choice for $S$. Fix $\chi \in C^{\infty}(\mathbb{R})$ that is positive on $(3,4)$ and supported in $[3,4]$. Let

$$
S(r)=-A \sum_{k=0}^{\infty} 10^{-4 k} \chi\left(10^{-k} r\right),
$$

where $A>0$ is a large constant that we will fix later. Recall from (2.1) that, as $\lambda \rightarrow \infty$,

$$
\operatorname{area}_{g}\left(\Sigma_{(\xi, \lambda)}\right)=4 \pi \lambda^{2}+2 \pi F_{0}(|\xi|)+F_{\sigma}(\xi, \lambda)+o(1) .
$$

We choose $\xi \in \mathbb{R}^{3}$ with $2 \leq|\xi| \leq 9$ and $\lambda=10^{j}$ where $j \geq 1$ is a large integer. Using (2.3), we compute

$$
\begin{aligned}
\left.\frac{d}{d s}\right|_{s=1} \operatorname{area}_{g}\left(\Sigma_{(s \xi, \lambda)}\right) & =2 \pi|\xi| F_{0}^{\prime}(|\xi|)+\frac{1}{2} \int_{X \in B_{\lambda}(\lambda \xi)} R(X)\langle\xi, \lambda \xi-X\rangle+o(1) \\
& =2 \pi|\xi| F_{0}^{\prime}(|\xi|)-4 \int_{X \in B_{\lambda}(\lambda \xi)} S(|X|)\langle\xi, \lambda \xi-X\rangle+o(1) \\
& =2 \pi|\xi| F_{0}^{\prime}(|\xi|)+4 A \int_{Y \in B_{1}(\xi)} \chi(|Y|)\langle\xi, \xi-Y\rangle+o(1) .
\end{aligned}
$$

When $|\xi|=2 \sqrt{2}$, the integral on the last line is negative. We choose $A>0$ large so that the sum of the first two terms is negative. When $|\xi|=5$, the second term vanishes while the first term is strictly positive. Thus, for $j \geq 1$ sufficiently large, the derivative

$$
\left.\frac{d}{d s}\right|_{s=1} \operatorname{area}_{g}\left(\Sigma_{(s \xi, \lambda)}\right)
$$

is negative when $|\xi|=2 \sqrt{2}$ and positive when $|\xi|=5$. Using that the metric $g$ is rotationally symmetric, we see that the map

$$
\xi \mapsto \operatorname{area}_{g}\left(\Sigma_{\left(\xi, 10^{j}\right)}\right)
$$

has a stable critical point - a local minimum - at some $\xi_{j} \in \mathbb{R}^{3}$ with $\left|\xi_{j}\right| \in(2 \sqrt{2}, 5)$. In other words, $\Sigma_{\left(\xi_{j}, 10^{j}\right)}$ is a "far-off-center" stable constant mean sphere for $j$ sufficiently large.

Remark 3.2. As noted by S. Brendle in [1, Theorem 1.5], the work of F. Pacard and $\mathrm{X}$. Xu [12] shows that every rotationally symmetric Riemannian manifold whose scalar curvature has a strict local extremum contains small stable constant mean curvature spheres. 


\section{Proof of Theorem 1.5}

Consider

$$
g_{i j}=\left(1+|x|^{-1}\right)^{4} \delta_{i j}+\sigma_{i j}
$$

with

$$
\partial_{I} \sigma_{i j}=O\left(|x|^{-2-|I|}\right) \quad \text { as }|x| \rightarrow \infty
$$

for all multi-indices $I$ of length $|I| \leq 7$.

Our proof follows the Lyapunov-Schmidt reduction and the expansion for the reduced area functional in [3]. However, we need to extend the approach of [3] to allow for the transition $\xi \rightarrow \infty$. To this end, we develop the expansion of the reduced area functional to a higher order than was necessary in [3].

The computations in this section are also related and in part inspired by those for exact Schwarzschild in [2, Appendix A]. Note that part of our expansion for the reduced area functional $\operatorname{area}_{g}\left(\Sigma_{(\xi, \lambda)}\right)$ follows upon rescaling the chart at infinity by $\lambda|\xi|$ from the work of S. Nardulli [11] or of F. Pacard and X. Xu [12]. Note that the estimate for the error term in (1.6) in, e.g., [12] is $O\left(\lambda^{2}|\xi|^{-5}\right)$, where we obtain $O\left(\lambda^{-1}|\xi|^{-6}\right)+O\left(|\xi|^{-7}\right)$. Our stronger estimate is crucial for our applications in this paper.

Let $\lambda>0$ and $\xi \in \mathbb{R}^{3}$ both be large. There exist $r>1$ with $r \sim \lambda$ and a smooth function $u_{(\xi, \lambda)}$ on the sphere $S_{r}(\lambda \xi)$ that is perpendicular to constants and linear functions with respect to the Euclidean metric such that the mean curvature with respect to $g$ of the Euclidean normal graph $\Sigma_{(\xi, \lambda)}$ of $u_{(\xi, \lambda)}$ - as a function on $S_{r}(\lambda \xi)$ - is a linear combination of a constant and a linear function and such that

$$
\operatorname{vol}_{g}\left(\Sigma_{(\xi, \lambda)}\right)=\frac{4 \pi \lambda^{3}}{3}
$$

Moreover,

$$
\sup _{S_{r}(\lambda \xi)}\left|u_{(\xi, \lambda)}\right|+\lambda \sup _{S_{r}(\lambda \xi)}\left|\nabla u_{(\xi, \lambda)}\right|+\lambda^{2} \sup _{S_{r}(\lambda \xi)}\left|\nabla^{2} u_{(\xi, \lambda)}\right|=O\left(\frac{1}{|\xi|}\right) .
$$

Indeed, this is a standard consequence of the implicit function theorem; cf. [3, Proposition 4]. We will improve estimate (4.1) below.

It is convenient to abbreviate $a=\lambda \xi$. In this section, we will frequently use standard integral identities recalled in Appendix A.

4.1. Estimating $\operatorname{vol}_{g}\left(B_{r}(a)\right)$. We have that

$$
\begin{aligned}
&\left(1+|x|^{-1}\right)^{6} \sqrt{\operatorname{det}\left(\delta_{i j}+\left(1+|x|^{-1}\right)^{-4} \sigma_{i j}\right)} \\
&=\left(1+|x|^{-1}\right)^{6}+\frac{1}{2}\left(1+|x|^{-1}\right)^{2} \operatorname{tr} \sigma \\
& \quad+\frac{1}{4}\left(1+|x|^{-1}\right)^{-2}\left(\frac{1}{2}(\operatorname{tr} \sigma)^{2}-|\sigma|^{2}\right)+O\left(|x|^{-6}\right) .
\end{aligned}
$$

Noting the dependence of the error term on $r$ in [2, proof of Proposition 17], we find

$$
\begin{gathered}
\int_{B_{r}(a)}\left(1+|x|^{-1}\right)^{6}=\frac{4 \pi}{3} \\
r^{3}\left(1+|a|^{-1}\right)^{6}\left(1+3\left(1+|a|^{-1}\right)^{-2} \frac{r^{2}}{|a|^{4}}+\frac{9}{7} \frac{r^{4}}{|a|^{6}}\right) \\
+O\left(r^{8}|a|^{-7}\right) .
\end{gathered}
$$


Next, we turn to the terms in the expansion of the volume form (4.2) that involve $\sigma$. We write $\underline{\sigma}$ for $\sigma$ evaluated at $a$. When $\underline{\sigma}$ appears with derivatives, the derivatives are taken first, then the result is evaluated at $a$.

First, note that for $y \in B_{r}(0)$ with $x=a+y$,

$$
\begin{gathered}
\left(1+|x|^{-1}\right)^{2}=\left(1+|a|^{-1}\right)^{2}+2\left(1+|a|^{-1}\right)\left(|a+y|^{-1}-|a|^{-1}\right) \\
+\underbrace{\left(|a+y|^{-1}-|a|^{-1}\right)^{2}}_{=O\left(|a|^{-4}|y|^{2}\right)}
\end{gathered}
$$

as well as

$$
|a+y|^{-1}-|a|^{-1}=-\frac{\langle a, y\rangle}{|a|^{3}}-\frac{1}{2} \frac{|a|^{2}|y|^{2}-3\langle a, y\rangle^{2}}{|a|^{5}}+O\left(r^{3}|a|^{-4}\right) .
$$

By Taylor expansion, we have that

$$
\begin{aligned}
\operatorname{tr} \sigma=\operatorname{tr} \underline{\sigma} & +\nabla_{y} \operatorname{tr} \underline{\sigma}+\frac{1}{2} \nabla_{y, y}^{2} \operatorname{tr} \underline{\sigma}+\frac{1}{6} \nabla_{y, y, y}^{3} \operatorname{tr} \underline{\sigma} \\
& +\frac{1}{24} \nabla_{y, y, y, y}^{4} \operatorname{tr} \underline{\sigma}+O\left(|y|^{5}|x|^{-7}\right) .
\end{aligned}
$$

Combining the above expansions and using the expressions found in Appendix A, we have

$$
\begin{aligned}
& \frac{1}{2} \int_{B_{r}(a)}\left(1+|x|^{-1}\right)^{2} \operatorname{tr} \sigma=\frac{1}{2}(1\left.+|a|^{-1}\right)^{2} \int_{B_{r}(a)} \operatorname{tr} \sigma \\
&+\left(1+|a|^{-1}\right) \int_{B_{r}(a)}\left(|a+y|^{-1}-|a|^{-1}\right) \operatorname{tr} \sigma \\
&+O\left(r^{5}|a|^{-6}\right) \\
&=\frac{1}{2}\left(1+|a|^{-1}\right)^{2} \int_{B_{r}} \operatorname{tr} \underline{\sigma}+\frac{1}{4}\left(1+|a|^{-1}\right)^{2} \int_{B_{r}} \nabla_{y, y}^{2} \operatorname{tr} \underline{\sigma} \\
&+ \frac{1}{48}\left(1+|a|^{-1}\right)^{2} \int_{B_{r}} \nabla_{y, y, y, y}^{4} \operatorname{tr} \underline{\sigma} \\
&-\left(1+|a|^{-1}\right)|a|^{-3} \int_{B_{r}}\langle a, y\rangle \nabla_{y} \operatorname{tr} \underline{\sigma} \\
&+O\left(r^{5}|a|^{-6}\right)+O\left(r^{7}|a|^{-7}\right) \\
&=\frac{2 \pi}{3} r^{3}\left(1+|a|^{-1}\right)^{2} \operatorname{tr} \underline{\sigma}+\frac{\pi}{15} r^{5}\left(1+|a|^{-1}\right)^{2} \Delta \operatorname{tr} \underline{\sigma} \\
&+\frac{\pi}{420} r^{7} \Delta(\Delta \operatorname{tr} \underline{\sigma})-\frac{4 \pi}{15}\left(1+|a|^{-1}\right) r^{5}|a|^{-3} \nabla_{a} \operatorname{tr} \underline{\sigma} \\
&+O\left(r^{5}|a|^{-6}\right)+O\left(r^{7}|a|^{-7}\right) .
\end{aligned}
$$

We now turn to the third term in the expansion of the volume form (4.2),

$$
\begin{aligned}
& \frac{1}{4} \int_{B_{r}(a)}\left(1+|x|^{-1}\right)^{-2}\left(\frac{1}{2}(\operatorname{tr} \sigma)^{2}-|\sigma|^{2}\right) \\
& \quad=\frac{\pi}{3} r^{3}\left(1+|a|^{-1}\right)^{-2}\left(\frac{1}{2}(\operatorname{tr} \underline{\sigma})^{2}-|\underline{\sigma}|^{2}\right)+O\left(r^{5}|a|^{-6}\right) .
\end{aligned}
$$


Now, putting these terms together, we find that

$$
\begin{aligned}
\operatorname{vol}_{g}\left(B_{r}(a)\right)=\frac{4 \pi}{3} & r^{3}\left(1+|a|^{-1}\right)^{6}\left(1+3\left(1+|a|^{-1}\right)^{-2} \frac{r^{2}}{|a|^{4}}+\frac{9}{7} \frac{r^{4}}{|a|^{6}}\right) \\
& +\frac{2 \pi}{3} r^{3}\left(1+|a|^{-1}\right)^{2} \operatorname{tr} \underline{\sigma}+\frac{\pi}{15} r^{5}\left(1+|a|^{-1}\right)^{2} \Delta \operatorname{tr} \underline{\sigma} \\
& +\frac{\pi}{420} r^{7} \Delta(\Delta \operatorname{tr} \underline{\sigma})-\frac{4 \pi}{15}\left(1+|a|^{-1}\right) r^{5}|a|^{-3} \nabla_{a} \operatorname{tr} \underline{\sigma} \\
& +\frac{\pi}{3} r^{3}\left(1+|a|^{-1}\right)^{-2}\left(\frac{1}{2}(\operatorname{tr} \underline{\sigma})^{2}-|\underline{\sigma}|^{2}\right)+O\left(r^{5}|a|^{-6}\right)+O\left(r^{8}|a|^{-7}\right) .
\end{aligned}
$$

4.2. Estimating area $\left.\operatorname{ar}_{\boldsymbol{r}}(\boldsymbol{a})\right)$. The density of the area form of $S_{r}(a)$ relative to the Euclidean background is given by

$$
\begin{aligned}
&\left(1+|x|^{-1}\right)^{4} \sqrt{\operatorname{det}\left(\left.\delta\right|_{S}+\left.\left(1+|x|^{-1}\right)^{-4} \sigma\right|_{S}\right)} \\
&=\left(1+|x|^{-1}\right)^{4}+\frac{1}{2} \operatorname{tr}_{S} \sigma+\frac{1}{4}\left(1+|x|^{-1}\right)^{-4}\left(\frac{1}{2}\left(\operatorname{tr}_{S} \sigma\right)^{2}-\left.|\sigma| S\right|^{2}\right)+O\left(|x|^{-6}\right) \\
&=\left(1+|x|^{-1}\right)^{4}+\frac{1}{2} \operatorname{tr} \sigma-\frac{1}{2} r^{-2} \sigma(y, y) \\
& \quad+\frac{1}{4}\left(1+|x|^{-1}\right)^{-4}\left(\frac{1}{2}(\operatorname{tr} \sigma)^{2}-r^{-2}(\operatorname{tr} \sigma) \sigma(y, y)-|\sigma|^{2}\right. \\
&\left.\quad+2 r^{-2}|\sigma(y, \cdot)|^{2}-\frac{1}{2} r^{-4} \sigma(y, y)^{2}\right)+O\left(|x|^{-6}\right) .
\end{aligned}
$$

As in [2, Proposition 17], we have that

$$
\begin{gathered}
\int_{S_{r}(a)}\left(1+|x|^{-1}\right)^{4}=4 \pi r^{2}\left(1+|a|^{-1}\right)^{4}\left(1+2\left(1+|a|^{-1}\right)^{-2} \frac{r^{2}}{|a|^{4}}+\frac{6}{5} \frac{r^{4}}{|a|^{6}}\right) \\
+O\left(r^{7}|a|^{-7}\right) .
\end{gathered}
$$

We compute, using standard integral identities recalled in Appendix A,

$$
\begin{aligned}
\frac{1}{2} \int_{S_{r}(a)} \operatorname{tr} \sigma & =\frac{1}{2} \int_{S_{r}} \operatorname{tr} \underline{\sigma}+\frac{1}{4} \int_{S_{r}} \nabla_{y, y}^{2} \operatorname{tr} \underline{\sigma}+\frac{1}{48} \int_{S_{r}} \nabla_{y, y, y, y}^{4} \operatorname{tr} \underline{\sigma}+O\left(r^{8}|a|^{-8}\right) \\
& =2 \pi r^{2} \operatorname{tr} \underline{\sigma}+\frac{\pi}{3} r^{4} \Delta \operatorname{tr} \underline{\sigma}+\frac{\pi}{60} r^{6} \Delta(\Delta \operatorname{tr} \underline{\sigma})+O\left(r^{8}|a|^{-8}\right)
\end{aligned}
$$

and

$$
\begin{aligned}
\frac{1}{2} \int_{S_{r}(a)} r^{-2} \sigma(y, y)= & \frac{1}{2} \int_{S_{r}} r^{-2} \underline{\sigma}(y, y)+\frac{1}{4} \int_{S_{r}} r^{-2}\left(\nabla_{y, y}^{2} \underline{\sigma}\right)(y, y) \\
& +\frac{1}{48} \int_{S_{r}} r^{-2}\left(\nabla_{y, y, y, y}^{4} \underline{\sigma}\right)(y, y)+O\left(r^{8}|a|^{-8}\right) \\
= & \frac{2 \pi}{3} r^{2} \operatorname{tr} \underline{\sigma}+\frac{\pi}{15} r^{4} \Delta \operatorname{tr} \underline{\sigma}+\frac{2 \pi}{15} r^{4} \operatorname{div}(\operatorname{div}(\underline{\sigma})) \\
& +\frac{\pi}{420} r^{6} \Delta(\Delta \operatorname{tr} \underline{\sigma})+\frac{\pi}{105} \Delta\left(\operatorname{div}(\operatorname{div}(\underline{\sigma}))+O\left(r^{8}|a|^{-8}\right) .\right.
\end{aligned}
$$


Putting these two expressions together, we find

$$
\begin{aligned}
\frac{1}{2} \int_{S_{r}(a)} \operatorname{tr} \sigma-\frac{1}{2} \int_{S_{r}(a)} r^{-2} \sigma(y, y)=2 \pi r^{2} \operatorname{tr} \underline{\sigma}-\frac{2 \pi}{3} r^{2} \operatorname{tr} \underline{\sigma}+\frac{\pi}{3} r^{4} \Delta \operatorname{tr} \underline{\sigma} \\
-\frac{\pi}{15} r^{4} \Delta \operatorname{tr} \underline{\sigma}-\frac{2 \pi}{15} r^{4} \operatorname{div}(\operatorname{div}(\underline{\sigma})) \\
+\frac{\pi}{60} r^{6} \Delta(\Delta \operatorname{tr} \underline{\sigma})-\frac{\pi}{420} r^{6} \Delta(\Delta \operatorname{tr} \underline{\sigma}) \\
-\frac{\pi}{105} \Delta\left(\operatorname{div}(\operatorname{div}(\underline{\sigma}))+O\left(r^{8}|a|^{-8}\right)\right. \\
=\frac{4 \pi}{3} r^{2} \operatorname{tr} \underline{\sigma}+\frac{4 \pi}{15} r^{4} \Delta(\operatorname{tr} \underline{\sigma})-\frac{2 \pi}{15} r^{4} \operatorname{div}(\operatorname{div}(\underline{\sigma})) \\
+\frac{\pi}{70} r^{6} \Delta(\Delta \operatorname{tr} \underline{\sigma})-\frac{\pi}{105} \Delta(\operatorname{div}(\operatorname{div}(\underline{\sigma})) \\
+O\left(r^{8}|a|^{-8}\right) .
\end{aligned}
$$

Finally, we compute

$$
\begin{aligned}
\frac{1}{4} \int_{S_{r}(a)}\left(1+|x|^{-1}\right)^{-4}\left(\frac{1}{2}(\operatorname{tr} \sigma)^{2}-r^{-2}(\operatorname{tr} \sigma) \sigma(y, y)-|\sigma|^{2}\right. & \left.+2 r^{-2}|\sigma(y, \cdot)|^{2}-\frac{1}{2} r^{-4} \sigma(y, y)^{2}\right) \\
= & \frac{1}{8}\left(1+|a|^{-1}\right)^{-4} \int_{S_{r}}(\operatorname{tr} \underline{\sigma})^{2}-\frac{1}{4}\left(1+|a|^{-1}\right)^{-4} \int_{S_{r}} r^{-2}(\operatorname{tr} \underline{\sigma}) \underline{\sigma}(y, y) \\
& -\frac{1}{4}\left(1+|a|^{-1}\right)^{-4} \int_{S_{r}}|\underline{\sigma}|^{2}+\frac{1}{2}\left(1+|a|^{-1}\right)^{-4} \int_{S_{r}} r^{-2}|\underline{\sigma}(y, \cdot)|^{2} \\
& -\frac{1}{8}\left(1+|a|^{-1}\right)^{-4} \int_{S_{r}} r^{-4} \underline{\sigma}(y, y)^{2}+O\left(r^{4}|a|^{-6}\right) \\
= & \frac{\pi}{2} r^{2}\left(1+|a|^{-1}\right)^{-4}(\operatorname{tr} \underline{\sigma})^{2}-\frac{\pi}{3} r^{2}\left(1+|a|^{-1}\right)^{-4}(\operatorname{tr} \underline{\sigma})^{2} \\
& -\pi r^{2}\left(1+|a|^{-1}\right)^{-4}|\underline{\sigma}|^{2}+\frac{2 \pi}{3} r^{2}\left(1+|a|^{-1}\right)^{-4}|\underline{\sigma}|^{2} \\
& -\frac{\pi}{30} r^{2}\left(1+|a|^{-1}\right)^{-4}(\operatorname{tr} \underline{\sigma})^{2}-\frac{\pi}{15} r^{2}\left(1+|a|^{-1}\right)^{-4}|\underline{\sigma}|^{2}+O\left(r^{4}|a|^{-6}\right) \\
= & \frac{2 \pi}{15} r^{2}\left(1+|a|^{-1}\right)^{-4}(\operatorname{tr} \underline{\sigma})^{2}-\frac{2 \pi}{5} r^{2}\left(1+|a|^{-1}\right)^{-4}|\underline{\sigma}|^{2}+O\left(r^{4}|a|^{-6}\right) \\
= & -\frac{2 \pi}{5} r^{2}\left(1+|a|^{-1}\right)^{-4}|\underline{o}|^{2}+O\left(r^{4}|a|^{-6}\right) .
\end{aligned}
$$

In conclusion, we have

$$
\begin{aligned}
\operatorname{area}_{g}\left(S_{r}(a)\right)=4 & \pi r^{2}\left(1+|a|^{-1}\right)^{4}\left(1+2\left(1+|a|^{-1}\right)^{-2} \frac{r^{2}}{|a|^{4}}+\frac{6}{5} \frac{r^{4}}{|a|^{6}}\right) \\
& +\frac{4 \pi}{3} r^{2} \operatorname{tr} \underline{\sigma}+\frac{4 \pi}{15} r^{4} \Delta \operatorname{tr} \underline{\sigma}-\frac{2 \pi}{15} r^{4} \operatorname{div}(\operatorname{div}(\underline{\sigma})) \\
& +\frac{\pi}{70} r^{6} \Delta(\Delta \operatorname{tr} \underline{\sigma})-\frac{\pi}{105} r^{6} \Delta(\operatorname{div}(\operatorname{div}(\underline{\sigma})) \\
& -\frac{2 \pi}{5} r^{2}\left(1+|a|^{-1}\right)^{-4}|\underline{o}|^{2}+O\left(r^{4}|a|^{-6}\right)+O\left(r^{7}|a|^{-7}\right) .
\end{aligned}
$$


4.3. Estimating $\mathscr{F}\left(S_{r}(a)\right)$. We let

$$
\mathscr{F}\left(S_{r}(a)\right)=\operatorname{area}_{g}\left(S_{r}(a)\right)-2 r^{-1}\left(1+|a|^{-1}\right)^{-2} \operatorname{vol}_{g}\left(S_{r}(a)\right) .
$$

We then compute

$$
\begin{aligned}
\mathscr{F}\left(S_{r}(a)\right)=4 \pi & r^{2}\left(1+|a|^{-1}\right)^{4}\left(1+2\left(1+|a|^{-1}\right)^{-2} \frac{r^{2}}{|a|^{4}}+\frac{6}{5} \frac{r^{4}}{|a|^{6}}\right) \\
& +\frac{4 \pi}{3} r^{2} \operatorname{tr} \underline{\sigma}+\frac{4 \pi}{15} r^{4} \Delta \operatorname{tr} \underline{\sigma}-\frac{2 \pi}{15} r^{4} \operatorname{div}(\operatorname{div}(\underline{\sigma})) \\
& +\frac{\pi}{70} r^{6} \Delta(\Delta \operatorname{tr} \underline{\sigma})-\frac{\pi}{105} r^{6} \Delta\left(\operatorname{div}(\operatorname{div}(\underline{\sigma}))-\frac{2 \pi}{5} r^{2}\left(1+|a|^{-1}\right)^{-4}|\underline{o}|^{2}\right. \\
& -\frac{8 \pi}{3} r^{2}\left(1+|a|^{-1}\right)^{4}\left(1+3\left(1+|a|^{-1}\right)^{-2} \frac{r^{2}}{|a|^{4}}+\frac{9}{7} \frac{r^{4}}{|a|^{6}}\right) \\
& -\frac{4 \pi}{3} r^{2} \operatorname{tr} \underline{\sigma}-\frac{2 \pi}{15} r^{4} \Delta \operatorname{tr} \underline{\sigma}-\frac{\pi}{210} r^{6} \Delta(\Delta \operatorname{tr} \underline{\sigma}) \\
& +\frac{8 \pi}{15}\left(1+|a|^{-1}\right)^{-1} r^{4}|a|^{-3} \nabla_{a} \operatorname{tr} \underline{\sigma} \\
& -\frac{2 \pi}{3} r^{2}\left(1+|a|^{-1}\right)^{-4}\left(\frac{1}{2}(\operatorname{tr} \underline{\sigma})^{2}-|\underline{\sigma}|^{2}\right) \\
& +O\left(r^{4}|a|^{-6}\right)+O\left(r^{7}|a|^{-7}\right) \\
=\frac{4 \pi}{3} & r^{2}\left(1+|a|^{-1}\right)^{4}+\frac{48 \pi}{35} \frac{r^{6}}{|a|^{6}}+\frac{2 \pi}{15} r^{4} \Delta \operatorname{tr} \underline{\sigma}-\frac{2 \pi}{15} r^{4} \operatorname{div}(\operatorname{div}(\underline{\sigma})) \\
& +\frac{\pi}{105} r^{6} \Delta(\Delta \operatorname{tr} \underline{\sigma})-\frac{\pi}{105} r^{6} \Delta\left(\operatorname{div}(\operatorname{div}(\underline{\sigma}))-\frac{2 \pi}{5} r^{2}\left(1+|a|^{-1}\right)^{-4}|\underline{o}|^{2}\right. \\
& -\frac{2 \pi}{3} r^{2}\left(1+|a|^{-1}\right)^{-4}\left(\frac{1}{2}(\operatorname{tr} \underline{\sigma})^{2}-|\underline{\sigma}|^{2}\right) \\
& +\frac{8 \pi}{15} r^{4}|a|^{-3} \nabla_{a} \operatorname{tr}^{\sigma}+O^{2}\left(r^{4}|a|^{-6}\right)+O\left(r^{7}|a|^{-7}\right) . \\
&
\end{aligned}
$$

\subsection{Estimating the mean curvature of $S_{r}(a)$. Consider}

$$
\hat{g}_{i j}=\bar{g}_{i j}+\hat{\sigma}_{i j}
$$

where

$$
\hat{\sigma}_{i j}=\left(1+|x|^{-1}\right)^{-4} \sigma_{i j} .
$$

By the computation in [8, p. 418], we have

$$
\begin{aligned}
& \hat{H}=H-r^{-1} \operatorname{tr}_{S} \hat{\sigma}+r^{-3} \hat{\sigma}(y, y)-r^{-1} \operatorname{tr}_{S}(\nabla . \hat{\sigma})(y, \cdot) \\
&+\frac{1}{2} r^{-1} \operatorname{tr}_{S} \nabla_{y} \hat{\sigma}+O\left(r^{-1}|x|^{-4}\right) \\
&=2 r^{-1}-r^{-1} \operatorname{tr} \hat{\sigma}+2 r^{-3} \hat{\sigma}(y, y)-r^{-1} \operatorname{div}(\hat{\sigma})(y)+\frac{1}{2} r^{-1} \nabla_{y} \operatorname{tr} \hat{\sigma} \\
& \quad+\frac{1}{2} r^{-3}\left(\nabla_{y} \hat{\sigma}\right)(y, y)+O\left(r^{-1}|x|^{-4}\right)
\end{aligned}
$$

for the mean curvature of $S_{r}(a)$ with respect to $\hat{g}$. The mean curvature of $S_{r}(a)$ with respect 
to $g$ is given by

$$
\begin{aligned}
H_{g}=\left(1+|x|^{-1}\right)^{-2} \hat{H}-4\left(1+|x|^{-1}\right)^{-3}|x|^{-3}\langle x, \hat{v}\rangle \\
=\left(1+|x|^{-1}\right)^{-2} \hat{H}-4 r^{-1}\left(1+|x|^{-1}\right)^{-3}|x|^{-3}\langle x, y\rangle+O\left(|x|^{-4}\right) \\
=2 r^{-1}\left(1+|x|^{-1}\right)^{-2}-4 r^{-1}\left(1+|x|^{-1}\right)^{-3}|x|^{-3}\langle x, y\rangle \\
\quad-r^{-1}\left(1+|x|^{-1}\right)^{-2} \operatorname{tr} \hat{\sigma}+2 r^{-3}\left(1+|x|^{-1}\right)^{-2} \hat{\sigma}(y, y) \\
\quad-r^{-1}\left(1+|x|^{-1}\right)^{-2} \operatorname{div}(\hat{\sigma})(y)+\frac{1}{2} r^{-1}\left(1+|x|^{-1}\right)^{-2} \nabla_{y} \operatorname{tr} \hat{\sigma} \\
\quad+\frac{1}{2} r^{-3}\left(1+|x|^{-1}\right)^{-2}\left(\nabla_{y} \hat{\sigma}\right)(y, y)+O\left(|x|^{-4}\right) \\
=2 r^{-1}\left(1+|x|^{-1}\right)^{-2}-4 r^{-1}\left(1+|x|^{-1}\right)^{-3}|x|^{-3}\langle x, y\rangle \\
\quad-r^{-1}\left(1+|a|^{-1}\right)^{-6} \operatorname{tr} \sigma+2 r^{-3}\left(1+|a|^{-1}\right)^{-6} \sigma(y, y) \\
\quad-r^{-1} \operatorname{div}(\sigma)(y)+\frac{1}{2} r^{-1} \nabla_{y} \operatorname{tr} \sigma+\frac{1}{2} r^{-3}\left(\nabla_{y} \sigma\right)(y, y)+O\left(|x|^{-4}\right)
\end{aligned}
$$

Expanding as in [2, proof of Lemma 18], we have that

$$
\begin{aligned}
& \left(1+|x|^{-1}\right)^{-2}-2\left(1+|x|^{-1}\right)^{-3}|x|^{-3}\langle x, y\rangle \\
& \quad=\left(1+|a|^{-1}\right)^{-2}-\frac{|a|^{2}|y|^{2}-3\langle a, y\rangle^{2}}{|a|^{5}}+O\left(r^{3}|x|^{-4}\right) .
\end{aligned}
$$

Thus,

$$
\begin{aligned}
H_{g}=2 r^{-1} & \left(1+|a|^{-1}\right)^{-2}-2 r^{-1} \frac{|a|^{2}|y|^{2}-3\langle a, y\rangle^{2}}{|a|^{5}} \\
& -r^{-1}\left(1+|a|^{-1}\right)^{-6} \operatorname{tr} \sigma+2 r^{-3}\left(1+|a|^{-1}\right)^{-6} \sigma(y, y) \\
& -r^{-1} \operatorname{div}(\sigma)(y)+\frac{1}{2} r^{-1} \nabla_{y} \operatorname{tr} \sigma+\frac{1}{2} r^{-3}\left(\nabla_{y} \sigma\right)(y, y)+O\left(r^{2}|x|^{-4}\right) .
\end{aligned}
$$

Now, we consider the (Euclidean) projection of $H_{g}$ to $\Lambda_{2}$ and $\Lambda_{>2}$, where $\Lambda_{2}$ is the space of second eigenfunctions on $S_{r}$ and $\Lambda_{>2}$ is the $L^{2}\left(S_{r}\right)$-orthogonal complement of $\Lambda_{0} \oplus \Lambda_{1} \oplus \Lambda_{2}$ :

$$
\begin{aligned}
\operatorname{proj}_{\Lambda_{2}} H_{g}= & -\frac{2}{r} \frac{|a|^{2}|y|^{2}-3\langle a, y\rangle^{2}}{|a|^{5}}+2 r^{-3}\left(1+|a|^{-1}\right)^{-6} \operatorname{proj}_{\Lambda_{2}} \underline{\sigma}(y, y)+O\left(r^{2}|x|^{-4}\right) \\
=- & \frac{2}{r} \frac{|a|^{2}|y|^{2}-3\langle a, y\rangle^{2}}{|a|^{5}}+2 r^{-3}\left(1+|a|^{-1}\right)^{-6}\left(\underline{\sigma}(y, y)-\frac{1}{3}|y|^{2} \operatorname{tr} \underline{\sigma}\right) \\
& \quad+O\left(r^{2}|x|^{-4}\right) .
\end{aligned}
$$

For the higher eigenspaces, we will only need the estimate

$$
\operatorname{proj}_{\Lambda_{>2}} H_{g}=O\left(|x|^{-3}\right)+O\left(r^{2}|x|^{-4}\right) .
$$

4.5. Estimates for $\boldsymbol{u}$. Our goal here is to improve upon the initial estimate (4.1). To this end, let $t \in[0,1]$. Consider the Euclidean graph above $S_{r}(a)$ of the function $t u$. The initial normal speed with respect to $g$ of this family of surfaces is given by

$$
w=u g\left(\frac{y}{r}, v_{g}\right)
$$


Note that

$$
\begin{aligned}
g\left(\frac{y}{r}, v_{g}\right) & =\left(1+|x|^{-1}\right)^{2}+\frac{1}{2}\left(1+|x|^{-1}\right)^{-2} r^{-2} \sigma(y, y)+O\left(|x|^{-4}\right) \\
& =\left(1+|x|^{-1}\right)^{2}+O\left(|x|^{-2}\right)
\end{aligned}
$$

up to and including second derivatives. It follows that

$$
\Delta_{g}^{S_{r}(a)} w+\left(\left|h_{g}\right|_{g}^{2}+\operatorname{Ric}_{g}\left(v_{g}, v_{g}\right)\right) w=H_{g}-H_{g}^{\Sigma}+O\left(\lambda^{-3}|\xi|^{-2}\right),
$$

where $H_{g}$ is the mean curvature of $S_{r}(a)$ with respect to $g$. From this, we see that

$$
\Delta^{S_{r}(a)} u+2 r^{-2} u=H_{g}-H_{g}^{\Sigma}+O\left(\lambda^{-3}|\xi|^{-2}\right)
$$

In conjunction with the estimate

$$
\operatorname{proj}_{\Lambda_{>1}}\left(H_{g}-H_{g}^{\Sigma}\right)=\operatorname{proj}_{\Lambda_{>1}} H_{g}=O\left(\lambda^{-3}|\xi|^{-2}\right)+O\left(\lambda^{-2}|\xi|^{-3}\right)
$$

from the previous subsection, we obtain

$$
\sup _{S_{r}(\lambda \xi)}\left|u_{(\xi, \lambda)}\right|+\lambda \sup _{S_{r}(\lambda \xi)}\left|\nabla u_{(\xi, \lambda)}\right|+\lambda^{2} \sup _{S_{r}(\lambda \xi)}\left|\nabla^{2} u_{(\xi, \lambda)}\right|=O\left(\lambda^{-1}|\xi|^{-2}\right)+O\left(|\xi|^{-3}\right) .
$$

This allows us to improve the coarse estimate above to

$$
\Delta_{g}^{S_{r}(a)} w+\left(\left|h_{g}\right|_{g}^{2}+\operatorname{Ric}_{g}\left(v_{g}, v_{g}\right)\right) w=H_{g}-H_{g}^{\Sigma}+O\left(\lambda^{-5}|\xi|^{-4}\right)+O\left(\lambda^{-3}|\xi|^{-6}\right)
$$

Thus

$$
\Delta_{g}^{S_{r}(a)} w=\left(1+|a|^{-1}\right)^{-2} \Delta^{S_{r}(a)} u+O\left(\lambda^{-4}|\xi|^{-4}\right)+O\left(\lambda^{-3}|\xi|^{-5}\right)
$$

Continuing on, we have that

$$
\left|h_{g}\right|_{g}^{2}=2 r^{-2}\left(1+|a|^{-1}\right)^{-4}+O\left(\lambda^{-3}|\xi|^{-3}\right)+O\left(\lambda^{-4}|\xi|^{-2}\right)
$$

and

$$
\operatorname{Ric}_{g}\left(v_{g}, v_{g}\right)=O\left(\lambda^{-3}|\xi|^{-3}\right) \text {. }
$$

Putting these estimates together, we find that

$$
\begin{aligned}
& \left(1+|a|^{-1}\right)^{-2} \Delta^{S_{r}(a)} u+2 r^{-2}\left(1+|a|^{-1}\right)^{-2} u \\
& \quad=H_{g}-H_{g}^{\Sigma}+O\left(\lambda^{-4}|\xi|^{-4}\right)+O\left(\lambda^{-3}|\xi|^{-5}\right)
\end{aligned}
$$

Hence,

$$
\begin{aligned}
\Delta & S_{r}(a) \\
& \operatorname{proj}_{\Lambda_{2}} u+2 r^{-2} \operatorname{proj}_{\Lambda_{2}} u \\
& =\operatorname{proj}_{\Lambda_{2}}\left(\Delta^{S_{r}(a)} u+2 r^{-2} u\right) \\
& =\left(1+|a|^{-1}\right)^{2} \operatorname{proj}_{\Lambda_{2}} H_{g}+O\left(\lambda^{-4}|\xi|^{-4}\right)+O\left(\lambda^{-3}|\xi|^{-5}\right) \\
& =-\frac{2}{r} \frac{|a|^{2}|y|^{2}-3\langle a, y\rangle^{2}}{|a|^{5}}+\frac{2}{r^{3}}\left(1+|a|^{-1}\right)^{-4}\left(\underline{\sigma}(y, y)-\frac{1}{3}|y|^{2} \operatorname{tr} \underline{\sigma}\right)+O\left(\lambda^{-2}|\xi|^{-4}\right) .
\end{aligned}
$$


This implies that

$$
\operatorname{proj}_{\Lambda_{2}} u=\frac{r}{2} \frac{|a|^{2}|y|^{2}-3\langle a, y\rangle^{2}}{|a|^{5}}-\frac{1}{2 r}\left(1+|a|^{-1}\right)^{-4}\left(\underline{\sigma}(y, y)-\frac{1}{3}|y|^{2} \operatorname{tr} \underline{\sigma}\right)+O\left(|\xi|^{-4}\right)
$$

together with two derivatives. In particular,

$$
\operatorname{proj}_{\Lambda_{2}} u=O\left(\lambda^{-1}|\xi|^{-2}\right)+O\left(|\xi|^{-3}\right) .
$$

The above expression also implies that

$$
\operatorname{proj}_{\Lambda_{>2}} u=O\left(\lambda^{-1}|\xi|^{-3}\right)+O\left(|\xi|^{-4}\right)
$$

along with two derivatives.

\subsection{Estimating $\mathscr{F}(\Sigma)$. Let}

$$
\mathscr{F}(\Sigma)=\operatorname{area}_{g}(\Sigma)-2 r^{-1}\left(1+|a|^{-1}\right)^{-2} \operatorname{vol}_{g}(\Omega),
$$

where $\Omega$ is the compact region bounded by $\Sigma$. Recall that

$$
\operatorname{vol}_{g}(\Omega)=\frac{4 \pi}{3} \lambda^{3} .
$$

Our goal is to compare $\mathscr{F}(\Sigma)$ with $\mathscr{F}\left(S_{r}(a)\right)$. We will make heavy use of the estimates for $u$ derived in the previous subsection. Computing as in [3, proof of Proposition 5], we obtain

$$
\begin{aligned}
\mathscr{F}(\Sigma)=\mathscr{F} & \left(S_{r}(a)\right)+\int_{S_{r}(a)}\left(H_{g}-2 r^{-1}\left(1+|a|^{-1}\right)^{-2}\right) w d \mu_{g} \\
& +\frac{1}{2} \int_{S_{r}(a)} H_{g}\left(H_{g}-2 r^{-1}\left(1+|a|^{-1}\right)^{-2}\right) w^{2} d \mu_{g} \\
& -\frac{1}{2} \int_{S_{r}(a)}\left(\Delta_{g}^{S_{r}(a)} w+\left(\left|h_{g}\right|_{g}^{2}+\operatorname{Ric}_{g}\left(v_{g}, v_{g}\right)\right) w\right) w d \mu_{g} \\
& +O\left(\lambda^{-4}|\xi|^{-6}\right)+O\left(\lambda^{-1}|\xi|^{-9}\right) .
\end{aligned}
$$

By the computation at the beginning of Section 4.2,

$$
d \mu_{g}=\left(\left(1+|x|^{-1}\right)^{4}+\frac{1}{2} \operatorname{tr} \sigma-\frac{1}{2} r^{-2} \sigma(y, y)+O\left(|x|^{-4}\right)\right) d \mu_{\bar{g}} .
$$

We begin with the first of three terms in the expansion of $\mathscr{F}(\Sigma)$ off of $\mathscr{F}\left(S_{r}(a)\right)$.

$$
\begin{aligned}
& \int_{S_{r}(a)}\left(H_{g}-2 r^{-1}\left(1+|a|^{-1}\right)^{-2}\right) w d \mu_{g} \\
& =\int_{S_{r}(a)}\left(H_{g}-2 r^{-1}\left(1+|a|^{-1}\right)^{-2}\right) u\left(1+|x|^{-1}\right)^{6}+O\left(\lambda^{-3}|\xi|^{-7}\right)+O\left(\lambda^{-2}|\xi|^{-8}\right) \\
& =\left(1+|a|^{-1}\right)^{6} \int_{S_{r}(a)}\left(H_{g}-2 r^{-1}\left(1+|a|^{-1}\right)^{-2}\right) u+O\left(\lambda^{-2}|\xi|^{-7}\right)+O\left(\lambda^{-1}|\xi|^{-8}\right) \\
& =-2 r^{-1}\left(1+|a|^{-1}\right)^{6} \int_{S_{r}(a)}\left(\frac{|a|^{2}|y|^{2}-3\langle a, y\rangle^{2}}{|a|^{5}}\right) u+2 r^{-3} \int_{S_{r}(a)} \underline{\sigma}(y, y) u \\
& \quad+O\left(\lambda^{-1}|\xi|^{-6}\right)+O\left(|\xi|^{-7}\right)
\end{aligned}
$$




$$
\begin{aligned}
=- & \int_{S_{r}}\left(\frac{|a|^{2}|y|^{2}-3\langle a, y\rangle^{2}}{|a|^{5}}\right)^{2}+r^{-2}\left(1+|a|^{-1}\right)^{2} \int_{S_{r}}\left(\frac{|a|^{2}|y|^{2}-3\langle a, y\rangle^{2}}{|a|^{5}}\right) \underline{\sigma}(y, y) \\
& -r^{-4}\left(1+|a|^{-1}\right)^{-4} \int_{S_{r}}\left(\underline{\sigma}(y, y)-\frac{1}{3}|y|^{2} \operatorname{tr} \underline{\sigma}\right)^{2} \\
& +r^{-2} \int_{S_{r}}\left(\frac{|a|^{2}|y|^{2}-3\langle a, y\rangle^{2}}{|a|^{5}}\right) \underline{\sigma}(y, y)+O\left(\lambda^{-1}|\xi|^{-6}\right)+O\left(|\xi|^{-7}\right) \\
=- & \int_{S_{r}}\left(\frac{|a|^{2}|y|^{2}-3\left\langle a,\left.y\right|^{2}\right.}{|a|^{5}}\right)^{2}+2 r^{-2} \int_{S_{r}}\left(\frac{|a|^{2}|y|^{2}-3\langle a, y\rangle^{2}}{|a|^{5}}\right) \underline{\sigma}(y, y) \\
& -r^{-4}\left(1+|a|^{-1}\right)^{-4} \int_{S_{r}}\left(\underline{\sigma}(y, y)-\frac{1}{3}|y|^{2} \operatorname{tr} \underline{\sigma}\right)^{2}+O\left(\lambda^{-1}|\xi|^{-6}\right)+O\left(|\xi|^{-7}\right) .
\end{aligned}
$$

The second term satisfies

$$
\frac{1}{2} \int_{S_{r}(a)} H_{g}\left(H_{g}-2 r^{-1}\left(1+|a|^{-1}\right)^{-2}\right) w^{2} d \mu_{g}=O\left(\lambda^{-4}|\xi|^{-6}\right)+O\left(\lambda^{-1}|\xi|^{-9}\right)
$$

Finally, the last term satisfies

$$
\begin{aligned}
& -\frac{1}{2} \int_{S_{r}(a)}\left(\Delta_{g}^{S_{r}(a)} w+\left(\left|h_{g}\right|_{g}^{2}+\operatorname{Ric}_{g}\left(v_{g}, v_{g}\right)\right) w\right) w d \mu_{g} \\
& =-\frac{1}{2}\left(1+|a|^{-1}\right)^{4} \int_{S_{r}(a)}\left(\Delta^{S_{r}} u+2 r^{-2} u\right) u+O\left(\lambda^{-3}|\xi|^{-6}\right)+O\left(\lambda^{-1}|\xi|^{-8}\right) \\
& =2 r^{-2}\left(1+|a|^{-1}\right)^{4} \int_{S_{r}(a)}\left(\operatorname{proj}_{\Lambda_{2}} u\right)^{2}+O\left(\lambda^{-2}|\xi|^{-6}\right)+O\left(|\xi|^{-8}\right) \\
& =\frac{1}{2} r^{-4}\left(1+|a|^{-1}\right)^{-4} \int_{S_{r}}\left(\underline{\sigma}(y, y)-\frac{1}{3}|y|^{2} \operatorname{tr} \underline{\sigma}\right)^{2} \\
& \quad-r^{-2} \int_{S_{r}}\left(\frac{|a|^{2}|y|^{2}-3\langle a, y\rangle^{2}}{|a|^{5}}\right) \underline{\sigma}(y, y)+\frac{1}{2} \int_{S_{r}}\left(\frac{|a|^{2}|y|^{2}-3\langle a, y\rangle^{2}}{|a|^{5}}\right)^{2} \\
& \quad+O\left(\lambda^{-1}|\xi|^{-6}\right)+O\left(|\xi|^{-7}\right) .
\end{aligned}
$$

Putting this together, we find that

$$
\begin{aligned}
\mathscr{F}(\Sigma)=\mathscr{F} & \left(S_{r}(a)\right)-\int_{S_{r}}\left(\frac{|a|^{2}|y|^{2}-3\langle a, y\rangle^{2}}{|a|^{5}}\right)^{2} \\
& +2 r^{-2} \int_{S_{r}}\left(\frac{|a|^{2}|y|^{2}-3\langle a, y\rangle^{2}}{|a|^{5}}\right) \underline{\sigma}(y, y) \\
& -r^{-4}\left(1+|a|^{-1}\right)^{-4} \int_{S_{r}}\left(\underline{\sigma}(y, y)-\frac{1}{3}|y|^{2} \operatorname{tr} \underline{\sigma}\right)^{2} \\
& +\frac{1}{2} r^{-4}\left(1+|a|^{-1}\right)^{-4} \int_{S_{r}}\left(\underline{\sigma}(y, y)-\frac{1}{3}|y|^{2} \operatorname{tr} \underline{\sigma}\right)^{2} \\
& -r^{-2} \int_{S_{r}}\left(\frac{|a|^{2}|y|^{2}-3\langle a, y\rangle^{2}}{|a|^{5}}\right) \underline{\sigma}(y, y) \\
& +\frac{1}{2} \int_{S_{r}}\left(\frac{|a|^{2}|y|^{2}-3\langle a, y\rangle^{2}}{|a|^{5}}\right)^{2}+O\left(\lambda^{-1}|\xi|^{-6}\right)+O\left(|\xi|^{-7}\right)
\end{aligned}
$$




$$
\begin{aligned}
=\mathscr{F} & \left(S_{r}(a)\right)-\frac{1}{2} \int_{S_{r}}\left(\frac{|a|^{2}|y|^{2}-3\langle a, y\rangle^{2}}{|a|^{5}}\right)^{2} \\
& +r^{-2} \int_{S_{r}}\left(\frac{|a|^{2}|y|^{2}-3\langle a, y\rangle^{2}}{|a|^{5}}\right) \underline{\sigma}(y, y) \\
& -\frac{1}{2} r^{-4}\left(1+|a|^{-1}\right)^{-4} \int_{S_{r}}\left(\underline{\sigma}(y, y)-\frac{1}{3}|y|^{2} \operatorname{tr} \underline{\sigma}\right)^{2} \\
& +O\left(\lambda^{-1}|\xi|^{-6}\right)+O\left(|\xi|^{-7}\right) .
\end{aligned}
$$

We now use the expansions given in Appendix A.3 to compute

$$
\begin{aligned}
\mathscr{F}(\Sigma)=\mathscr{F} & \left(S_{r}(a)\right)-\frac{8 \pi}{5}|\xi|^{-6}+\frac{8 \pi}{15} \frac{r^{4}}{|a|^{3}}\left(\operatorname{tr} \underline{\sigma}-3|a|^{-1} \underline{\sigma}(a, a)\right) \\
& -\frac{4 \pi}{15} r^{2}\left(1+|a|^{-1}\right)^{-4}|\underline{\sigma}|^{2}+O\left(\lambda^{-1}|\xi|^{-6}\right)+O\left(|\xi|^{-7}\right) \\
=\frac{4 \pi}{3} & r^{2}\left(1+|a|^{-1}\right)^{4}+\frac{48 \pi}{35} \frac{r^{6}}{|a|^{6}}+\frac{2 \pi}{15} r^{4} \Delta \operatorname{tr} \underline{\sigma}-\frac{2 \pi}{15} r^{4} \operatorname{div}(\operatorname{div}(\underline{\sigma})) \\
& +\frac{\pi}{105} r^{6} \Delta(\Delta \operatorname{tr} \underline{\sigma})-\frac{\pi}{105} r^{6} \Delta(\operatorname{div}(\operatorname{div}(\underline{\sigma})) \\
& -\frac{2 \pi}{5} r^{2}\left(1+|a|^{-1}\right)^{-4}|\underline{o}|^{2}-\frac{2 \pi}{3} r^{2}\left(1+|a|^{-1}\right)^{-4}\left(\frac{1}{2}(\operatorname{tr} \underline{\sigma})^{2}-|\underline{\sigma}|^{2}\right) \\
& +\frac{8 \pi}{15} r^{4}|a|^{-3} \nabla_{a} \operatorname{tr} \underline{\sigma}-\frac{8 \pi}{5}|\xi|^{-6}+\frac{8 \pi}{15} \frac{r^{4}}{|a|^{3}}\left(\operatorname{tr} \underline{\sigma}-3|a|^{-1} \underline{\sigma}(a, a)\right) \\
& -\frac{4 \pi}{15} r^{2}\left(1+|a|^{-1}\right)^{-4}|\underline{o}|^{2}+O\left(\lambda^{-1}|\xi|^{-6}\right)+O\left(|\xi|^{-7}\right) \\
= & \frac{4 \pi}{3} r^{2}\left(1+|a|^{-1}\right)^{4}-\frac{8 \pi}{35}|\xi|^{-6}+\frac{2 \pi}{15} r^{4} \Delta \operatorname{tr} \underline{\sigma}-\frac{2 \pi}{15} r^{4} \operatorname{div}(\operatorname{div}(\underline{\sigma})) \\
& +\frac{\pi}{105} r^{6} \Delta(\Delta \operatorname{tr} \underline{\sigma})-\frac{\pi}{105} r^{6} \Delta\left(\operatorname{div}(\operatorname{div}(\underline{\sigma}))-\frac{2 \pi}{3} r^{2}\left(1+|a|^{-1}\right)^{-4}|\underline{\sigma}|^{2}\right. \\
& -\frac{2 \pi}{3} r^{2}\left(1+|a|^{-1}\right)^{-4}\left(\frac{1}{2}(\operatorname{tr} \underline{\sigma})^{2}-|\underline{\sigma}|^{2}\right)+\frac{8 \pi}{15} r^{4}|a|^{-3} \nabla_{a} \operatorname{tr} \underline{\sigma} \\
& +\frac{8 \pi}{15} \frac{r^{4}}{|a|^{3}}\left(\operatorname{tr} \underline{\sigma}-3|a|^{-1} \underline{\sigma}(a, a)\right)+O\left(\lambda{ }^{-1}|\xi|^{-6}\right)+O\left(|\xi|^{-7}\right) .
\end{aligned}
$$

Going back to the definition of $\mathscr{F}(\Sigma)$, we obtain that

$$
\begin{aligned}
\operatorname{area}_{g}(\Sigma)=\frac{4 \pi}{3} & r^{2}\left(1+|a|^{-1}\right)^{4}+\frac{8 \pi}{3} \lambda^{3} r^{-1}\left(1+|a|^{-1}\right)^{-2}-\frac{8 \pi}{35}|\xi|^{-6} \\
& +\frac{2 \pi}{15} r^{4} \Delta \operatorname{tr} \underline{\sigma}-\frac{2 \pi}{15} r^{4} \operatorname{div}(\operatorname{div}(\underline{\sigma}))+\frac{\pi}{105} r^{6} \Delta(\Delta \operatorname{tr} \underline{\sigma}) \\
& -\frac{\pi}{105} r^{6} \Delta\left(\operatorname{div}(\operatorname{div}(\underline{\sigma}))-\frac{2 \pi}{3} r^{2}\left(1+|a|^{-1}\right)^{-4}|\underline{\sigma}|^{2}\right. \\
& -\frac{2 \pi}{3} r^{2}\left(1+|a|^{-1}\right)^{-4}\left(\frac{1}{2}(\operatorname{tr} \underline{\sigma})^{2}-|\underline{\sigma}|^{2}\right)+\frac{8 \pi}{15} r^{4}|a|^{-3} \nabla_{a}(\operatorname{tr} \underline{\sigma}) \\
& +\frac{8 \pi}{15} \frac{r^{4}}{|a|^{3}}\left(\operatorname{tr} \underline{\sigma}-3|a|^{-2} \underline{\sigma}(a, a)\right)+O\left(\lambda^{-1}|\xi|^{-6}\right)+O\left(|\xi|^{-7}\right) .
\end{aligned}
$$


4.7. Estimating $r$. We now use the expansion

$$
\begin{gathered}
\operatorname{vol}_{g}(\Omega)=\operatorname{vol}_{g}\left(B_{r}(a)\right)+\int_{S_{r}(a)} w d \mu_{g}+\frac{1}{2} \int_{S_{r}(a)} H_{g} w^{2} d \mu_{g} \\
+O\left(\lambda^{-3}|\xi|^{-6}\right)+O\left(|\xi|^{-9}\right)
\end{gathered}
$$

to relate $\lambda$ and $r$. Note that because $u$ is orthogonal to constants and to linear functions,

$$
\int_{S_{r}(a)} w d \mu_{g}=O\left(|\xi|^{-5}\right)+O\left(\lambda|\xi|^{-6}\right)
$$

and

$$
\frac{1}{2} \int_{S_{r}(a)} H_{g} w^{2} d \mu_{g}=O\left(\lambda^{-1}|\xi|^{-4}\right)+O\left(\lambda|\xi|^{-6}\right)
$$

Hence, using the expression for $\operatorname{vol}_{g}\left(B_{r}(a)\right)$ obtained previously, we find that

$$
\begin{aligned}
\frac{4 \pi}{3} \lambda^{3} & =\frac{4 \pi}{3} r^{3}\left(1+|a|^{-1}\right)^{6}+\frac{2 \pi}{3} r^{3}\left(1+|a|^{-1}\right)^{2} \operatorname{tr} \underline{\sigma}+O\left(\lambda|\xi|^{-4}\right) \\
& =\frac{4 \pi}{3} r^{3}\left(1+|a|^{-1}\right)^{6}\left(1+\frac{1}{2}\left(1+|a|^{-1}\right)^{-4} \operatorname{tr} \underline{\sigma}+O\left(\lambda^{-2}|\xi|^{-4}\right)\right) .
\end{aligned}
$$

It is convenient to write

$$
\lambda^{3}=r^{3}\left(1+|a|^{-1}\right)^{6}(1+\psi),
$$

where

$$
\psi=\frac{1}{2}\left(1+|a|^{-1}\right)^{-4} \operatorname{tr} \underline{\sigma}+O\left(\lambda^{-2}|\xi|^{-4}\right)=O\left(\lambda^{-2}|\xi|^{-2}\right) .
$$

We now estimate the first line in the expansion for $\operatorname{area}_{g}(\Sigma)$ obtained above.

$$
\begin{aligned}
& \frac{4 \pi}{3} r^{2}\left(1+|a|^{-1}\right)^{4}+\frac{8 \pi}{3} \lambda^{3} r^{-1}\left(1+|a|^{-1}\right)^{-2} \\
& \quad=\frac{4 \pi}{3} r^{2}\left(1+|a|^{-1}\right)^{4}+\frac{8 \pi}{3} r^{2}\left(1+|a|^{-1}\right)^{4}(1+\psi) \\
& \quad=4 \pi r^{2}\left(1+|a|^{-1}\right)^{4}\left(1+\frac{2}{3} \psi\right) \\
& \quad=4 \pi r^{2}\left(1+|a|^{-1}\right)^{4}(1+\psi)^{\frac{2}{3}}+\frac{4 \pi}{9} r^{2}\left(1+|a|^{-1}\right)^{4} \psi^{2}+O\left(r^{2} \psi^{3}\right) \\
& \quad=4 \pi \lambda^{2}+\frac{\pi}{9} r^{2}\left(1+|a|^{-1}\right)^{-4}(\operatorname{tr} \underline{\sigma})^{2}+O\left(\lambda^{-2}|\xi|^{-6}\right) .
\end{aligned}
$$

4.8. Concluding the estimate for $\operatorname{area}_{g}(\Sigma)$. Combining the results in the previous two subsections, we obtain that

$$
\begin{aligned}
\operatorname{area}_{g}(\Sigma)=4 \pi & \lambda^{2}-\frac{8 \pi}{35}|\xi|^{-6}+\frac{\pi}{9} r^{2}\left(1+|a|^{-1}\right)^{-4}(\operatorname{tr} \underline{\sigma})^{2}+\frac{2 \pi}{15} r^{4} \Delta \operatorname{tr} \underline{\sigma} \\
& -\frac{2 \pi}{15} r^{4} \operatorname{div}(\operatorname{div}(\underline{\sigma}))+\frac{\pi}{105} r^{6} \Delta(\Delta \operatorname{tr} \underline{\sigma})-\frac{\pi}{105} r^{6} \Delta(\operatorname{div}(\operatorname{div}(\underline{\sigma})) \\
& -\frac{2 \pi}{3} r^{2}\left(1+|a|^{-1}\right)^{-4}|\underline{o}|^{2}-\frac{2 \pi}{3} r^{2}\left(1+|a|^{-1}\right)^{-4}\left(\frac{1}{2}(\operatorname{tr} \underline{\sigma})^{2}-|\underline{\sigma}|^{2}\right) \\
& +\frac{8 \pi}{15} r^{4}|a|^{-3} \nabla_{a} \operatorname{tr} \underline{\sigma}+\frac{8 \pi}{15} \frac{r^{4}}{|a|^{3}}\left(\operatorname{tr} \underline{\sigma}-3|a|^{-2} \underline{\sigma}(a, a)\right) \\
& +O\left(\lambda^{-1}|\xi|^{-6}\right)+O\left(|\xi|^{-7}\right)
\end{aligned}
$$




$$
\begin{aligned}
=4 \pi & \lambda^{2}-\frac{8 \pi}{35}|\xi|^{-6}+\frac{2 \pi}{15} r^{4} \Delta \operatorname{tr} \underline{\sigma}-\frac{2 \pi}{15} r^{4} \operatorname{div}(\operatorname{div}(\underline{\sigma})) \\
& +\frac{\pi}{105} r^{6} \Delta(\Delta \operatorname{tr} \underline{\sigma})-\frac{\pi}{105} r^{6} \Delta(\operatorname{div}(\operatorname{div}(\underline{\sigma})) \\
& +\frac{8 \pi}{15} r^{4}|a|^{-3} \nabla_{a} \operatorname{tr} \underline{\sigma}+\frac{8 \pi}{15} \frac{r^{4}}{|a|^{3}}\left(\operatorname{tr} \underline{\sigma}-3|a|^{-2} \underline{\sigma}(a, a)\right) \\
& +O\left(\lambda^{-1}|\xi|^{-6}\right)+O\left(|\xi|^{-7}\right) \\
=4 \pi & \lambda^{2}-\frac{8 \pi}{35}|\xi|^{-6}+\frac{2 \pi}{15} \lambda^{4}\left(1+|a|^{-1}\right)^{-8}(\Delta \operatorname{tr} \underline{\sigma}-\operatorname{div}(\operatorname{div}(\underline{\sigma}))) \\
& +\frac{\pi}{105} \lambda^{6}(\Delta(\Delta \operatorname{tr} \underline{\sigma})-\Delta(\operatorname{div}(\operatorname{div}(\underline{\sigma}))) \\
& +\frac{8 \pi}{15} \lambda^{4}|a|^{-3} \nabla_{a} \operatorname{tr} \underline{\sigma}+\frac{8 \pi}{15} \frac{\lambda^{4}}{|a|^{3}}\left(\operatorname{tr} \underline{\sigma}-3|a|^{-2} \underline{\sigma}(a, a)\right) \\
+ & O\left(\lambda^{-1}|\xi|^{-6}\right)+O\left(|\xi|^{-7}\right) .
\end{aligned}
$$

4.9. Estimating $\boldsymbol{R}$ and $\boldsymbol{\Delta} \boldsymbol{R}$. We now relate this expression for $\operatorname{area}_{g}(\Sigma)$ to the scalar curvature $R$ of $(M, g)$. As with mean curvature, we first consider

$$
\hat{g}_{i j}=\bar{g}_{i j}+\hat{\sigma}_{i j}
$$

where

$$
\hat{\sigma}_{i j}=\left(1+|x|^{-1}\right)^{-4} \sigma_{i j} .
$$

Then

$$
R_{\hat{g}}=\operatorname{div} \operatorname{div} \hat{\sigma}-\Delta \operatorname{tr} \hat{\sigma}+O\left(|x|^{-6}\right) .
$$

Note that

$$
\operatorname{div} \hat{\sigma}=\left(1+|x|^{-1}\right)^{-4} \operatorname{div} \sigma+4\left(1+|x|^{-1}\right)^{-5}|x|^{-3} \sigma(x, \cdot)
$$

and

$$
\begin{aligned}
\operatorname{div} \operatorname{div} \hat{\sigma}=(1+ & \left.|x|^{-1}\right)^{-4} \operatorname{div} \operatorname{div} \sigma+4\left(1+|x|^{-1}\right)^{-5}|x|^{-3}(\operatorname{div} \sigma)(x) \\
& +20\left(1+|x|^{-1}\right)^{-6}|x|^{-6} \sigma(x, x)-12\left(1+|x|^{-1}\right)^{-5}|x|^{-5} \sigma(x, x) \\
& +4\left(1+|x|^{-1}\right)^{-5}|x|^{-3}(\operatorname{div} \sigma)(x)+4\left(1+|x|^{-1}\right)^{-5}|x|^{-3} \operatorname{tr} \sigma \\
=(1+ & \left.+|x|^{-1}\right)^{-4} \operatorname{div} \operatorname{div} \sigma+8|x|^{-3}(\operatorname{div} \sigma)(x) \\
& +4\left(1+|x|^{-1}\right)^{-5}|x|^{-3}\left(\operatorname{tr} \sigma-3|x|^{-2} \sigma(x, x)\right)+O\left(|x|^{-6}\right) .
\end{aligned}
$$

Similarly,

$$
\begin{aligned}
\Delta \operatorname{tr} \hat{\sigma} & =\Delta\left(\left(1+|x|^{-1}\right)^{-4} \operatorname{tr} \sigma\right) \\
& =\left(1+|x|^{-1}\right)^{-4} \Delta \operatorname{tr} \sigma+8\left(1+|x|^{-1}\right)^{-5}|x|^{-3} \nabla_{x} \operatorname{tr} \sigma+(\operatorname{tr} \sigma) \Delta\left(1+|x|^{-1}\right)^{-4} \\
& =\left(1+|x|^{-1}\right)^{-4} \Delta \operatorname{tr} \sigma+8|x|^{-3} \nabla_{x} \operatorname{tr} \sigma+O\left(|x|^{-6}\right) .
\end{aligned}
$$

Thus, we find that

$$
\begin{aligned}
R_{\hat{g}}=(1 & \left.+|x|^{-1}\right)^{-4}(\operatorname{div} \operatorname{div} \sigma-\Delta \operatorname{tr} \sigma)+4|x|^{-3}\left(\operatorname{tr} \sigma-3|x|^{-2} \sigma(x, x)\right) \\
& +8|x|^{-3}(\operatorname{div} \sigma)(x)-8|x|^{-3} \nabla_{x} \operatorname{tr} \sigma+O\left(|x|^{-6}\right) .
\end{aligned}
$$


It follows that

$$
\begin{aligned}
R=- & 8\left(1+|x|^{-1}\right)^{-5} \Delta_{\hat{g}}|x|^{-1}+\left(1+|x|^{-1}\right)^{-4} R_{\hat{g}} \\
=- & 8\left(1+|x|^{-1}\right)^{-5} \Delta_{\hat{g}}|x|^{-1}+\left(1+|x|^{-1}\right)^{-8}(\operatorname{div}(\operatorname{div}(\sigma))-\Delta \operatorname{tr} \sigma) \\
& +4|x|^{-3}\left(\operatorname{tr} \sigma-3|x|^{-2} \sigma(x, x)\right)+8|x|^{-3}(\operatorname{div} \sigma)(x) \\
& \quad-8|x|^{-3} \nabla_{x} \operatorname{tr} \sigma+O\left(|x|^{-6}\right) .
\end{aligned}
$$

It remains to estimate $\Delta_{\hat{g}}|x|^{-1}$. We have that

$$
\sqrt{\operatorname{det} \hat{g}_{i j}}=\sqrt{\operatorname{det}\left(\delta_{i j}+\hat{\sigma}_{i j}\right)}=1+\frac{1}{2} \operatorname{tr} \hat{\sigma}+O\left(|x|^{-4}\right)
$$

and

$$
\hat{g}^{i j}=\delta^{i j}-\hat{\sigma}^{i j}+O\left(|x|^{-4}\right)
$$

Thus,

$$
\begin{gathered}
\Delta_{\hat{g}}|x|^{-1}=-3|x|^{-5} \sigma(x, x)+|x|^{-3} \operatorname{tr} \sigma+|x|^{-3}(\operatorname{div} \sigma)(x) \\
-\frac{1}{2}|x|^{-3} \nabla_{x} \operatorname{tr} \sigma+O\left(|x|^{-6}\right) .
\end{gathered}
$$

It follows that

$$
\begin{aligned}
R=(1 & \left.+|x|^{-1}\right)^{-8}(\operatorname{div}(\operatorname{div}(\sigma))-\Delta \operatorname{tr} \sigma)-4|x|^{-3}\left(\operatorname{tr} \sigma-3|x|^{-2} \sigma(x, x)\right) \\
& -4|x|^{-3} \nabla_{x} \operatorname{tr} \sigma+O\left(|x|^{-6}\right) .
\end{aligned}
$$

Similarly,

$$
\Delta R=\Delta(\operatorname{div}(\operatorname{div}(\sigma))-\Delta \operatorname{tr} \sigma)+O\left(|x|^{-7}\right) .
$$

4.10. Reduced area-functional. We finally obtain that, for $\xi \in \mathbb{R}^{3}$ and $\lambda>0$ both large,

$$
\begin{gathered}
\operatorname{area}_{g}\left(\Sigma_{(\xi, \lambda)}\right)=4 \pi \lambda^{2}-\frac{2 \pi}{15} \lambda^{4} \underline{R}-\frac{\pi}{105} \lambda^{6} \Delta \underline{R}-\frac{8 \pi}{35}|\xi|^{-6} \\
+O\left(\lambda^{-1}|\xi|^{-6}\right)+O\left(|\xi|^{-7}\right),
\end{gathered}
$$

where $R$ is the scalar curvature of $(M, g)$ and

$$
\begin{aligned}
\underline{R} & =R(\lambda \xi), \\
\Delta \underline{R} & =(\Delta R)(\lambda \xi) .
\end{aligned}
$$

This completes the proof of Theorem 1.5. We also record here the first radial derivative

$$
\begin{aligned}
\left.\frac{d}{d s}\right|_{s=1} \operatorname{area}_{g}\left(\Sigma_{(s \xi, \lambda)}\right)= & \frac{2 \pi}{15} \lambda^{5}|\xi| \partial_{r} \underline{R}-\frac{\pi}{105} \lambda^{7}|\xi| \partial_{r} \Delta \underline{R}+\frac{48 \pi}{35}|\xi|^{-6} \\
& +O\left(\lambda^{-1}|\xi|^{-6}\right)+O\left(|\xi|^{-7}\right) \\
= & \frac{\pi}{105}\left(-14 \lambda^{5}|\xi| \partial_{r} \underline{R}-\lambda^{7}|\xi| \partial_{r} \Delta \underline{R}+144|\xi|^{-6}\right) \\
& +O\left(\lambda^{-1}|\xi|^{-6}\right)+O\left(|\xi|^{-7}\right) .
\end{aligned}
$$




\section{Proof of Corollary 1.7}

We assume that $(M, g)$ is $C^{6}$-asymptotically Schwarzschild in the sense that

$$
g_{i j}=\left(1+|x|^{-1}\right)^{4} \delta_{i j}+\sigma_{i j},
$$

where $\partial_{I} \sigma_{i j}=O\left(|x|^{-2-|I|}\right)$ for all multi-indices $I$ of length $|I| \leq 6$. We also assume that

$$
x^{i} x^{j} \partial_{i} \partial_{j} R \geq 0
$$

outside of a compact set. This condition integrates to yield

$$
x^{i} \partial_{i} R \leq 0 \quad \text { and } \quad R \geq 0
$$

We now consider a sequence of connected closed stable constant mean curvature surfaces $\Sigma_{k}$ with

$$
r_{0}\left(\Sigma_{k}\right) \rightarrow \infty, \quad \operatorname{area}_{g}\left(\Sigma_{k}\right) \rightarrow \infty, \quad r_{0}\left(\Sigma_{k}\right) H\left(\Sigma_{k}\right) \rightarrow \infty .
$$

For $k$ large, we may find $\lambda>0$ and $\xi \in \mathbb{R}^{3}$ both large so that $\Sigma_{k}=\Sigma_{(\xi, \lambda)}$ and

$$
\left.\frac{d}{d s}\right|_{s=1} \operatorname{area}_{g}\left(\Sigma_{(s \xi, \lambda)}\right)=0 .
$$

By (4.3), we have that

$$
0=\frac{\pi}{105}\left(-14 \lambda^{5}|\xi| \partial_{r} \underline{R}-\lambda^{7}|\xi| \partial_{r} \Delta \underline{R}+144|\xi|^{-6}\right)+O\left(\lambda^{-1}|\xi|^{-6}\right)+O\left(|\xi|^{-7}\right) .
$$

It follows that

$$
\partial_{r} \underline{R}=O\left(\lambda^{-5}|\xi|^{-7}\right)=o\left(\lambda^{-5}|\xi|^{-5}\right) .
$$

Using this and (1.7), we may integrate in the radial direction to find that for $t \geq 0$,

$$
\left(\partial_{r} R\right)((1+t) \lambda \xi) \geq \partial_{r} \underline{R}=o\left(\lambda^{-5}|\xi|^{-5}\right) .
$$

Integrating this again, we find that

$$
\underline{R} \leq o\left(\lambda^{-4}|\xi|^{-4}\right) t+R((1+t) \lambda \xi) \leq O\left(\lambda^{-4}|\xi|^{-4}\right)\left(o(1) t+(1+t)^{-4}\right) .
$$

Choosing $t$ judiciously, we arrange for the term in parenthesis to be $o(1)$. We have proven that

$$
\underline{R}=o\left(\lambda^{-4}|\xi|^{-4}\right) \text {. }
$$

On the other hand, by combining $\partial_{r} \underline{R}=o\left(\lambda^{-5}|\xi|^{-5}\right), \partial_{r} R \leq 0$, and Taylor's theorem, we find

$$
\partial_{r}^{2} \underline{R}=o\left(\lambda^{-6}|\xi|^{-6}\right) .
$$

Together with (1.7), we similarly obtain

$$
\partial_{r}^{3} \underline{R}=o\left(\lambda^{-7}|\xi|^{-7}\right) .
$$

Now, we consider the first variation of $\operatorname{area}_{g}\left(\Sigma_{(\xi, \lambda)}\right)$ in directions orthogonal to $\xi$. It follows that

$$
D \underline{R}=O\left(\lambda^{-5}|\xi|^{-7}\right)
$$


Now, as in the radial case, combining Taylor's theorem with $R \geq 0, \underline{R}=o\left(\lambda^{-4}|\xi|^{-4}\right)$, and $D \underline{R}=o\left(\lambda^{-5}|\xi|^{-5}\right)$, we obtain

$$
D^{2} \underline{R} \geq-o\left(\lambda^{-6}|\xi|^{-6}\right)
$$

Similarly, we find that

$$
D^{2} \partial_{r} \underline{R} \leq o\left(\lambda^{-7}|\xi|^{-7}\right)
$$

Finally, we see from

$$
\partial_{r} \Delta \underline{R}=\Delta \partial_{r} \underline{R}-2|\xi|^{-1} \lambda^{-1} \Delta \underline{R}+2 \lambda^{-1}|\xi|^{-1} \partial_{r}^{2} \underline{R}+2 \lambda^{-2}|\xi|^{-2} \partial_{r} \underline{R}
$$

that

$$
\partial_{r} \Delta \underline{R} \leq o\left(\lambda^{-7}|\xi|^{-7}\right) .
$$

Returning to the radial first variation, we see that

$$
0 \geq 14 \lambda^{5}|\xi| \partial_{r} \underline{R} \geq 144|\xi|^{-6}+O\left(\lambda^{-1}|\xi|^{-6}\right)+O\left(|\xi|^{-7}\right) .
$$

This contradiction completes the proof.

\section{Proof of Theorem 1.8}

Our strategy here follows that of [3, proof of Theorem 1]. However, unlike in [3], we work in the regime where $\xi \rightarrow \infty$.

Let $S:(0, \infty) \rightarrow(-\infty, 0]$ be a smooth function with

$$
S^{(\ell)}=O\left(r^{-5-\ell}\right)
$$

for every non-negative integer $\ell$. We define a smooth function $\varphi:(0, \infty) \rightarrow \mathbb{R}$ by

$$
\varphi(r)=\frac{1}{r} \int_{r}^{\infty}(\rho-r) \rho S(\rho) d \rho .
$$

Arguing as in Lemma 3.1, we find that

$$
\varphi^{(\ell)}(r)=O\left(r^{-5-\ell}\right)
$$

for all derivatives. We now define a conformally flat Riemannian metric

$$
g=\left(1+\frac{1}{r}+\varphi(r)\right)^{4} \bar{g}=\left(1+\frac{1}{r}\right)^{4} \bar{g}+O\left(\frac{1}{r^{5}}\right)
$$

on the complement of a compact subset of $\mathbb{R}^{3}$. Note that the asymptotics of $g$ are of the form asserted in Theorem 1.8. The scalar curvature $R$ of $g$ satisfies

$$
R=-8\left(1+O\left(\frac{1}{r}\right)\right) S(r) .
$$

Fix $\chi \in C^{\infty}(\mathbb{R})$ with support in $[4,6]$ that is positive on $(4,6)$. Assume that $\chi^{\prime}(5)=-1$. Define

$$
S(r)=-\sum_{j=0}^{\infty} 10^{-5 j} \chi\left(10^{-j} r\right) .
$$

Note that, as required, $S^{(\ell)}(r)=O\left(r^{-5-\ell}\right)$. 
Consider $\xi \in \mathbb{R}^{3}$ with $|\xi|=10^{k} t$ for $t \in[3,7]$. Then, taking $\lambda=10^{k}$, we have that

$$
\begin{aligned}
\operatorname{area}_{g}\left(\Sigma_{\left(\xi, 10^{k}\right)}\right)= & 4 \pi \lambda^{2}-\frac{2 \pi}{15} 10^{4 k} R\left(10^{k} \xi\right)-\frac{\pi}{105} 10^{6 k}(\Delta R)\left(10^{k} \xi\right) \\
& \quad-\frac{8 \pi}{35}|\xi|^{-6}+O\left(10^{-7 k}\right) \\
= & 4 \pi \lambda^{2}+\frac{16 \pi}{15} 10^{-6 k} \chi(t)-\frac{8 \pi}{35} 10^{-6 k} t^{-6}+O\left(10^{-7 k}\right)
\end{aligned}
$$

and

$$
\left.\frac{d}{d s}\right|_{s=1} \operatorname{area}_{g}\left(\Sigma_{\left(s \xi, 10^{k}\right)}\right)=\frac{16 \pi}{15} 10^{-6 k} \chi^{\prime}(t)+\frac{48 \pi}{35} 10^{-6 k} t^{-6}+O\left(10^{-7 k}\right) .
$$

For $t=7$, we have

$$
\left.\frac{d}{d s}\right|_{s=1} \operatorname{area}_{g}\left(\Sigma_{\left(s \xi, 10^{k}\right)}\right)=\frac{48 \pi}{35} 10^{-6 k} 7^{-6}+O\left(10^{-7 k}\right)>0
$$

for sufficiently large $k$. On the other hand, for $t=5$, we have

$$
\left.\frac{d}{d s}\right|_{s=1} \operatorname{area}_{g}\left(\Sigma_{\left(s \xi, 10^{k}\right)}\right)=-\frac{16 \pi}{15} 10^{-6 k}+\frac{48 \pi}{35} 10^{-6 k} 7^{-6}+O\left(10^{-7 k}\right)<0 .
$$

It follows that for some $t_{k} \in(5,7)$ and every $\xi_{k} \in \mathbb{R}^{3}$ with $\left|\xi_{k}\right|=10^{k} t_{k}, \Sigma_{\left(\xi_{k}, 10^{k}\right)}$ is a stable constant mean curvature sphere. This completes the proof.

\section{A. Some integral expressions}

In this appendix, we recall several standard identities that are used in the proof of Theorem 1.5. A comprehensive account of computations of this type is given in the book [7] by A. Gray. See in particular [7, Appendix A.2] for the calculation of the moments of the sphere.

A.1. Integrals over $B_{r}(0)$. Recall that

$$
\int_{B_{r}(0)}\left(y^{i}\right)^{2}=\frac{1}{3} \int_{B_{r}(0)}|y|^{2}=\frac{4 \pi}{15} r^{5} \quad \text { for all } i=1,2,3 .
$$

Thus, for a symmetric tensor $A_{i j}$ on $\mathbb{R}^{3}$, we have

$$
\sum_{i, j} \int_{B_{r}(0)} A_{i j} y^{i} y^{j}=\frac{4 \pi}{15} r^{5} \operatorname{tr} A .
$$

Similarly,

$$
\int_{B_{r}(0)}\left(y^{i}\right)^{4}=\frac{4 \pi}{35} r^{7}
$$

and for $i \neq j$,

$$
\int_{B_{r}(0)}\left(y^{i}\right)^{2}\left(y^{j}\right)^{2}=\frac{4 \pi}{105} r^{7} .
$$


For a totally symmetric tensor $B_{i j k l}$ on $\mathbb{R}^{3}$, we have that

$$
\begin{aligned}
\sum_{i, j, k, l} \int_{B_{r}(0)} B_{i j k l} y^{i} y^{j} y^{k} y^{l} & =\sum_{i} B_{i i i i} \int_{B_{r}(0)}\left(y^{i}\right)^{4}+3 \sum_{i \neq j} B_{i i j j} \int_{B_{r}(0)}\left(y^{i}\right)^{2}\left(y^{j}\right)^{2} \\
& =\frac{4 \pi}{35} r^{7}\left(\sum_{i} B_{i i i i}+\sum_{i \neq j} B_{i i j j}\right) \\
& =\frac{4 \pi}{35} r^{7} \sum_{i, j} B_{i i j j} .
\end{aligned}
$$

A.2. Integrals over $S_{r}(0)$. Recall that

$$
\int_{S_{r}(0)}\left(y^{i}\right)^{2}=\frac{4 \pi}{3} r^{4} .
$$

It follows that, for a symmetric tensor $A_{i j}$ on $\mathbb{R}^{3}$,

$$
\sum_{i, j} \int_{S_{r}(0)} A_{i j} y^{i} y^{j}=\frac{4 \pi}{3} r^{4} \operatorname{tr} A .
$$

Similarly,

$$
\begin{aligned}
& \int_{S_{r}(0)}\left(y^{i}\right)^{4}=\frac{4 \pi}{5} r^{6} \quad \text { for all } i=1,2,3, \\
& \int_{S_{r}(0)}\left(y^{i}\right)^{2}\left(y^{j}\right)^{2}=\frac{4 \pi}{15} r^{6} \text { for all } i \neq j .
\end{aligned}
$$

Thus, for a totally symmetric tensor $B_{i j k l}$ on $\mathbb{R}^{3}$, we have

$$
\sum_{i, j, k, l} \int_{S_{r}(0)} B_{i j k l} y^{i} y^{j} y^{k} y^{l}=\frac{4 \pi}{5} r^{6} \sum_{i, j} B_{i i j j} .
$$

If $B_{i j k l}$ is symmetric in the first two slots and in the second two slots separately, we obtain

$$
\begin{aligned}
\sum_{i, j, k, l} \int_{S_{r}(0)} B_{i j k l} y^{i} y^{j} y^{k} y^{l}= & \sum_{i} B_{i i i i} \int_{S_{r}(0)}\left(y^{i}\right)^{4}+\sum_{i} \sum_{j \neq i} B_{i i j j} \int_{S_{r}(0)}\left(y^{i}\right)^{2}\left(y^{j}\right)^{2} \\
& +2 \sum_{i} \sum_{j \neq i} B_{i j i j} \int_{S_{r}(0)}\left(y^{i}\right)^{2}\left(y^{j}\right)^{2} \\
= & \frac{4 \pi}{15} r^{6}\left(3 \sum_{i} B_{i i i i}+\sum_{i} \sum_{j \neq i} B_{i i j j}+2 \sum_{i} \sum_{i \neq j} B_{i j i j}\right) \\
= & \frac{4 \pi}{15} r^{6}\left(\sum_{i, j} B_{i i j j}+2 \sum_{i, j} B_{i j i j}\right) .
\end{aligned}
$$

Finally, recall that

$$
\begin{aligned}
\int_{S_{r}(0)}\left(y^{i}\right)^{6} & =\frac{4 \pi}{7} r^{8} \quad \text { for all } i=1,2,3, \\
\int_{S_{r}(0)}\left(y^{i}\right)^{4}\left(y^{j}\right)^{2} & =\frac{4 \pi}{35} r^{8} \quad \text { when } i \neq j, \\
\int_{S_{r}(0)}\left(y^{1}\right)^{2}\left(y^{2}\right)^{2}\left(y^{3}\right)^{2} & =\frac{4 \pi}{105} r^{8} .
\end{aligned}
$$


Assume now that the tensor $C_{i j k l m n}$ on $\mathbb{R}^{3}$ is symmetric in the first four indices and, separately, in the last two indices.

$$
\begin{aligned}
& \sum_{i, j, k, l, m, n} \int_{S_{r}(0)} C_{i j k l m n} y^{i} y^{j} y^{k} y^{l} y^{m} y^{n} \\
& =\sum_{i} C_{i i i i i i} \int_{S_{r}(0)}\left(y^{i}\right)^{6}+6 \sum_{\substack{i, j \\
\text { distinct }}} C_{i i j j j j} \int_{S_{r}(0)}\left(y^{i}\right)^{2}\left(y^{j}\right)^{4} \\
& \quad+\sum_{\substack{i, j \\
\text { distinct }}} C_{i i i i j j} \int_{S_{r}(0)}\left(y^{i}\right)^{4}\left(y^{j}\right)^{2}+3 \sum_{i, j, k} C_{i i j j k k} \int_{S_{r}(0)}\left(y^{i}\right)^{2}\left(y^{j}\right)^{2}\left(y^{k}\right)^{2} \\
& \quad+8 \sum_{\substack{i, j \\
\text { distinct }}} C_{i i i j i j} \int_{S_{r}(0)}\left(y^{i}\right)^{4}\left(y^{j}\right)^{2}+12 \sum_{i, j, k} C_{i i j k j k} \int_{S_{r}(0)}\left(y^{i}\right)^{2}\left(y^{j}\right)^{2}\left(y^{k}\right)^{2} \\
& =\frac{4 \pi}{35} r^{8}\left(\sum_{i, j, k} C_{i i j j k k}+4 \sum_{i, j, k} C_{i i j k j k}\right) .
\end{aligned}
$$

A.3. Further useful integrals. The following computations needed in the proof of Theorem 1.5 are readily verified using the identities from the previous subsection. Recall that $\underline{\sigma}$ is a constant tensor field of rank 2 .

$$
\begin{aligned}
\int_{S_{r}(0)}\left(\frac{|a|^{2}|y|^{2}-3\langle a, y\rangle^{2}}{|a|^{5}}\right)^{2} & =\frac{16 \pi}{5} \frac{r^{6}}{|a|^{6}}, \\
\int_{S_{r}(0)}\left(\underline{\sigma}(y, y)-\frac{1}{3}|y|^{2} \operatorname{tr} \underline{\sigma}\right)\left(\frac{|a|^{2}|y|^{2}-3\langle a, y\rangle^{2}}{|a|^{5}}\right) & =\int_{S_{r}(0)} \underline{\sigma}(y, y)\left(\frac{|a|^{2}|y|^{2}-3\langle a, y\rangle^{2}}{|a|^{5}}\right) \\
& =\frac{8 \pi}{15} \frac{r^{6}}{|a|^{3}}\left(\operatorname{tr} \underline{\sigma}-3|a|^{-2} \underline{\sigma}(a, a)\right), \\
\int_{S_{r}(0)}\left(\underline{\sigma}(y, y)-\frac{1}{3}|y|^{2} \operatorname{tr} \underline{\sigma}\right)^{2} & =\frac{8 \pi}{45} r^{6}\left(3|\underline{\sigma}|^{2}-(\operatorname{tr} \underline{\sigma})^{2}\right) \\
& =\frac{8 \pi}{15} r^{6}|\underline{\underline{\sigma}}|^{2} .
\end{aligned}
$$

\section{References}

[1] S. Brendle, Constant mean curvature surfaces in warped product manifolds, Publ. Math. Inst. Hautes Études Sci. 117 (2013), 247-269.

[2] S. Brendle and M. Eichmair, Isoperimetric and Weingarten surfaces in the Schwarzschild manifold, J. Differential Geom. 94 (2013), no. 3, 387-407.

[3] S. Brendle and M. Eichmair, Large outlying stable constant mean curvature spheres in initial data sets, Invent. Math. 197 (2014), no. 3, 663-682.

[4] A. Carlotto, O. Chodosh and M. Eichmair, Effective versions of the positive mass theorem, Invent. Math. 206 (2016), no. 3, 975-1016.

[5] O. Chodosh and M. Eichmair, Global uniqueness of large stable CMC surfaces in asymptotically flat Riemannian 3-manifolds, preprint 2017, https: //arxiv .org/abs/1703. 02494.

[6] M. Eichmair and J. Metzger, On large volume preserving stable CMC surfaces in initial data sets, J. Differential Geom. 91 (2012), no. 1, 81-102. 
[7] A. Gray, Tubes. With a preface by Vicente Miquel, 2nd ed., Progr. Math. 221, Birkhäuser, Basel 2004.

[8] G. Huisken and T. Ilmanen, The inverse mean curvature flow and the Riemannian Penrose inequality, J. Differential Geom. 59 (2001), no. 3, 353-437.

[9] G. Huisken and S.-T. Yau, Definition of center of mass for isolated physical systems and unique foliations by stable spheres with constant mean curvature, Invent. Math. 124 (1996), no. 1-3, 281-311.

[10] S. Ma, Unstable CMC spheres and outlying CMC spheres in AF 3-manifolds, Commun. Contemp. Math. 20 (2018), no. 1, Article ID 1650065.

[11] S. Nardulli, The isoperimetric profile of a smooth Riemannian manifold for small volumes, Ann. Global Anal. Geom. 36 (2009), no. 2, 111-131.

[12] F. Pacard and $X . X u$, Constant mean curvature spheres in Riemannian manifolds, Manuscripta Math. 128 (2009), no. 3, 275-295.

[13] J. Qing and G. Tian, On the uniqueness of the foliation of spheres of constant mean curvature in asymptotically flat 3-manifolds, J. Amer. Math. Soc. 20 (2007), no. 4, 1091-1110.

[14] R. Ye, Foliation by constant mean curvature spheres, Pacific J. Math. 147 (1991), no. 2, 381-396.

Otis Chodosh, Department of Mathematics, Princeton University,

Fine Hall, Washington Road, Princeton, NJ 08544, USA

Current address: Department of Mathematics, Stanford University, Building 380, Stanford, CA 94305, USA https://orcid.org/0000-0002-6124-7889

e-mail: ochodosh@stanford.edu

Michael Eichmair, Faculty of Mathematics, University of Vienna,

Oskar-Morgenstern-Platz 1, 1090 Vienna, Austria

https://orcid.org/0000-0001-7993-9536

e-mail: michael.eichmair@univie.ac.at

Eingegangen 31. August 2018 